九州大学学術情報リポジトリ

Kyushu University Institutional Repository

An iterative method for obtaining a nonlinear solution for the temperature distribution of a rotating spherical body revolving in a circular orbit around a star

Sekiya, Minoru

Department of Earth and Planetary Sciences, Faculty of Sciences, Kyushu University : Professor

Shimoda, Akihito

Graduate School of Science, Kyushu University

http://hdl. handle. net/2324/1448921

出版情報: Planetary and Space Science. 84, pp.112-121，2013-07-05. Pergamon Press バージョン：

権利関係 : 


\title{
An iterative method for obtaining a nonlinear solution for the temperature distribution of a rotating spherical body revolving in a circular orbit around a star
}

\author{
M. Sekiya*, A. A. Shimoda \\ Department of Earth and Planetary Sciences, Faculty of Sciences, 33 Kyushu University, \\ Hakozaki, Fukuoka 812-8581, Japan
}

\begin{abstract}
We developed an iterative method for determining the time-dependent threedimensional temperature distribution in a spherical body with smooth surface that is irradiated by a star. In the method developed in our previous paper (Sekiya et al., 2012), only the rotational motion is taken into account and the effect due to the revolution around the star is ignored. The present work includes both the effects of the rotation and the revolution. We take into account the cooling due to the surface radiation that is proportional to the fourth power of the temperature; this is the difference in the present work from Vokrouhlický (1999) that employs the linear approximation for the radiative cooling. It is assumed that material parameters such as the thermal conductivity and the thermometric conductivity are constant throughout the spherical body. We obtain a general solution for the temperature distribution inside a body by using the spherical harmonics and the spherical Bessel func-
\end{abstract}

\footnotetext{
*Corresponding author. Phone: +81 92642 2669. Fax: +81 926422684.

Email addresses: sekiya.minoru.393@m.kyushu-u.ac.jp (M. Sekiya), shimoda.akihito@geo.kyushu-u.ac.jp (A. A. Shimoda) 
tions for space and the Fourier series for the time. The term in the boundary condition that represents the heating due to the star is also expanded into the spherical harmonics and the Fourier series. The coefficients of the general solution are fitted to satisfy the surface boundary condition by using an iterative method. We obtained solutions that satisfy the nonlinear boundary condition within $0.1 \%$ accuracy. The temperature distribution determined according to the iterative method is different from that according to the linear approximation; both the maximum and minimum temperatures at a given time after the summer solstice for an iterative solution are lower than those for a linear solution. The maximum difference between rate of change of the semimajor axis due to the Yarkovsky effect according to the iterative solution and that according to the linear solution is about $20 \%$. Therefore, current understanding of the Yarkovsky effect based on linear solutions is fairly good.

Keywords: Temperature, radiation, asteroids, small bodies, meteorites

\section{Introduction}

In planetary science, accurate determination of the temperature distribution in a small body is important. The Yarkovsky effect, i.e. the variation of the orbital semimajor axis of a small body in a planetary system due to the recoil force of the thermal radiation, is determined by the surface temperature distribution and its diurnal and seasonal variation (Bottke et al., 2006, and reference therein). In many asteroid families, members with small absolute magnitude (i.e. large ones) reside near the mean value of the semimajor axis, while members with large absolute magnitude (i.e. small ones) 
spread over a wide range of the semimajor axis; this is explained by the Yarkovsky effect because a smaller body has a larger surface area-to-mass ratio and susceptible to the orbital change due to the surface recoil force of the thermal radiation (Vokrouhlický et al., 2006a,b). The Yarkovsky effect is also important for continuous supply of near-Earth asteroids whose survival time is considered much less than the age of the solar system (Gladman et al., 2000). The main belt asteroids migrate slowly by the Yarkovsky effect, and some of which are captured into, for example, powerful $\nu_{6}$ secular resonance at the inner edge of the asteroid belt and 3/1 mean-motion resonance, etc., and transported to near-Earth region (Bottke et al., 2002; Morbidelli and Vokrouhlický, 2003; La Spina et al., 2004).

Information on the albedo and size distribution of asteroids is important to elucidate their mineralogy, taxonomy and origins. Simultaneous observations of an asteroid by the visible and the thermal infrared combined with a suitable thermal model allow one to determine the diameter and the albedo (Delbó and Harris, 2002; Masiero et al., 2011).

For a sample-return mission, e.g. Hayabusa (MUSES-C) of JAXA that collected more than 1500 dust particles from Asteroid 25143 Itokawa (Nakamura et al., 2011), preliminary estimates of size and geometry of a target body by using a thermal model was important for planning (Sekiguchi et al., 2003). Hayabusa 2 of JAXA and OSIRIS-REx of NASA are sample-return missions, targets of which are asteroids 1999 JU3 and 1999 RQ36, respectively; preliminary estimates of their temperature as a function of the depth are very important because organic compounds may be destroyed under a high temperature at its surface and we have to know in advance the depth 
below which organic compounds keep their primitive compositions (Michel and Delbo, 2011; Delbo and Michel, 2011).

Most of theoretical works on the thermal evolution of parent bodies of meteorites have been performed with an assumption that the surface temperature is given by a constant average temperature (Miyamoto et al., 1981; Grimm and McSween, 1989; Ghosh and McSween, 1998; Young et al., 2003; Wakita and Sekiya, 2011). However, the surface temperature is, in reality, not constant, and changes with time due to the rotation and revolution of a parent body. Some types of carbonaceous chondrites (CI, CM and CR) contain solar-wind-implanted gases; hence, these meteorites came from the surface of their parent bodies (Bischoff et al., 2006). Volatile organic matters and some components of the rare gases may be lost if the surface temperature rises significantly above the average temperature. Thus it would be interesting to incorporate the diurnal as well as the seasonal variation of the surface temperature distributions into the thermal evolution model of parent bodies of meteorites.

If radiogenic heat sources are negligible, the temperature distribution is determined by the surface heating due to the irradiation of the star, the radiative cooling due to the Stefan-Boltzmann law that is proportional to the fourth-power of the temperature, and the heat conduction inside the body. A pioneering work by Vokrouhlický (1998) solved the temperature distribution in a small body analytically taking the rotation of the body but omitting the revolution around a star by using the linear approximation $\left(T_{a v}+\delta T\right)^{4} \approx T_{a v}^{4}+4 T_{a v}^{3} \delta T$ for the radiative cooling term, where $T_{a v}$ is the average temperature and $\delta T$ is the difference between the temperature and 
the average temperature. In the subsequent work, Vokrouhlický (1999) obtained an analytic solution that takes into account both the rotation and the revolution completely, assuming that the orbit of the body is circular and using the linear approximation. Vokrouhlický and Farinella (1999) developed a semianalytical-nonlinear theory of the seasonal Yarkovsky effect by assuming an axisymmetric temperature distribution.

We recently developed an iterative method for determining temperature distribution of a rotating spherical body using the fourth power of the temperature for the radiative cooling taking only the rotational motion into account but omitting the seasonal variation due to the revolution around a star (Sekiya et al., 2012, hereafter cited as Paper I). Paper I corresponds to a nonlinear version of Vokrouhlický (1998). Next step is to develop a nonlinear version of Vokrouhlický (1999); this is the subject of the present work.

\section{Basic equations}

\subsection{General formulation}

We consider a spherical body that rotates at a constant angular velocity $\tilde{\omega}_{\text {rot }}$ (hereafter, we use a tilde for dimensional variables in order to distinguish them from the corresponding dimensionless variables that will be introduced later). It is assumed that the orbit of the body is circular; therefore, the body revolves around the star with a constant angular velocity $\tilde{\omega}_{\text {rev }}=\left(\tilde{G} \tilde{M}_{*} / \tilde{a}^{3}\right)^{1 / 2}$, where $\tilde{G}$ is the universal gravitational constant, $\tilde{M}_{*}$ is the mass of the star, and $\tilde{a}$ is the semimajor axis. It is assumed that the luminosity of the star, $\tilde{L}_{*}$, remains constant; therefore, the stellar flux $\tilde{F}_{*}=\tilde{L}_{*} /\left(4 \pi \tilde{a}^{2}\right)$ will also remain constant. Further, we assume that the sur- 
face of the body is smooth and all relevant physical properties of a body, such as the absorption coefficient $\alpha=1-A$ where $A$ is the Bond albedo, and the emissivity $\epsilon$ of the surface, thermal conductivity $\tilde{k}_{T}$, and the thermometric conductivity $\tilde{\kappa}=\tilde{k}_{T} /(\tilde{\rho} \tilde{C})$, where $\tilde{\rho}$ is the material density and $\tilde{C}$ is the specific heat, are constant throughout the object and remain constant with time.

\section{Figure 1}

We use a coordinate system $(\tilde{x}, \tilde{y}, \tilde{z})$ with the origin at the center of the body (see Fig. 1); this coordinate system does not rotate with respect to the inertial system, even though a translational displacement occurs due to the body's orbital motion around the star; the $\tilde{z}$ axis is set in the direction of the rotational angular momentum of the body; the $\tilde{x}$ axis is set so that the direction of the star viewed from the body on the time of summer solstice is in the $\tilde{x}-\tilde{z}$ plane at an angle $\gamma$ from the $\tilde{x}$-axis, where $\gamma$ is the obliquity of the rotation axis of the body; finally, $\tilde{y}$-axis is determined so that $\tilde{x}, \tilde{y}$ and $\tilde{z}$ form a right-handed coordinate system. The orbital velocity of the body is in the opposite direction of the $\tilde{y}$-axis at the time of summer solstice.

\section{Figure 2}

The thermal conduction equation is expressed as follows:

$$
\frac{\partial \tilde{T}}{\partial \tilde{t}}+\tilde{\omega}_{r o t} \frac{\partial \tilde{T}}{\partial \phi}=\frac{\tilde{\kappa}}{\tilde{r}^{2}}\left\{\frac{\partial}{\partial \tilde{r}}\left(\tilde{r}^{2} \frac{\partial \tilde{T}}{\partial \tilde{r}}\right)+\left[\frac{1}{\sin \theta} \frac{\partial}{\partial \theta}\left(\sin \theta \frac{\partial \tilde{T}}{\partial \theta}\right)+\frac{1}{\sin ^{2} \theta} \frac{\partial^{2} \tilde{T}}{\partial \phi^{2}}\right]\right\} .
$$

Here, we use the spherical coordinate system $(\tilde{r}, \theta, \phi)$, the pole of which is in the direction of the rotation axis; $\theta$ is the colatitude and $\phi$ is the longitude 
measured from the $\tilde{x}-\tilde{z}$ plane as illustrated in Fig. 2, and $\tilde{r}$ is the distance from the center of the body. Note that $\tilde{r}=\tilde{R}$ at the surface of the body, where $\tilde{R}$ is the radius of the body. Further, $\tilde{T}$ is the temperature, $\tilde{t}$ is the time, and the second term on the left-hand side (LHS) represents the advection of the temperature for an angular velocity $\tilde{\omega}_{\text {rot }}$. In Paper I, the first term on the LHS was omitted, because we ignored the orbital motion of the body. On the other hand, we take into account the orbital motion in this paper, and the direction of stellar flux changes with time; hence, the first term on the LHS should remain. Because the orbital motion is periodic with the revolution period $\tilde{P}_{\text {rev }}=2 \pi / \tilde{\omega}_{\text {rev }}$, we restrict ourselves to solve solutions of $\tilde{T}$ that have the period $\tilde{P}_{r e v}$. Note that the rotational motion is treated as the advection, hence, the period of $\tilde{T}$ with respect to time is independent of the rotation period $\tilde{P}_{\text {rot }}=2 \pi / \tilde{\omega}_{\text {rot }}$; this is the advantage of treating the rotational motion as the advection in non-rotating frame of reference.

The stellar heating rate $\tilde{\Gamma}$ is given by Eq. (3) of Paper I, where $\Theta$ is the angle between the direction of the star and of a point with the spherical coordinates $(\tilde{R}, \theta, \phi)$ on the surface of the body when viewed from the center of the body, as shown in Fig. 2. Note that $\Theta$ is a function of $\theta, \phi$ and $\tilde{t}$, unlike the case of Paper I in which $\Theta$ was independent of $\tilde{t}$. The dependence of $\Theta$ on $\tilde{t}$ is periodic with the period $\tilde{P}_{r e v}$. The surface boundary condition is given by Eq. (4) of Paper I that expresses the energy balance of the radiative cooling, thermal conduction, and heating by the star per unit time and per unit surface area of a body. The total balance of the stellar heating rate and radiative cooling rate is expressed as:

$$
\pi \tilde{R}^{2} \alpha \tilde{F}_{*}=4 \pi \tilde{R}^{2} \epsilon \tilde{\sigma}<<<\tilde{T}^{4}>_{\phi}>_{\theta}>_{\tilde{t}}
$$


where $\tilde{\sigma}$ is the Stefan-Boltzmann constant, $<>_{\phi} \equiv[1 /(2 \pi)] \int_{-\pi}^{\pi} d \phi$ is the mean with respect to the longitude, $<>_{\theta} \equiv(1 / 2) \int_{-1}^{1} d \cos \theta$ is the mean with respect to the colatitude, and $<>_{\tilde{t}} \equiv\left(1 / \tilde{P}_{\text {rev }}\right) \int_{0}^{\tilde{P}_{\text {rev }}} d \tilde{t}$ is the mean with respect to the time. Note that the mean with respective to the time of the surface integral of the heat conduction flux must be zero because of the energy conservation. We define the average temperature as $\tilde{T}_{a v} \equiv\left[<<<\tilde{T}^{4}>_{\phi}>_{\theta}>_{\tilde{t}}\right.$ ]$^{1 / 4}=\left[\alpha \tilde{F}_{*} /(4 \epsilon \tilde{\sigma})\right]^{1 / 4}=280\left[(\alpha / \epsilon)\left(\tilde{L}_{*} / \tilde{L}_{\odot}\right)\right]^{1 / 4}(\tilde{a} / \mathrm{AU})^{-1 / 2}[\mathrm{~K}]$, where $\tilde{L}_{\odot}$ is the solar luminosity.

In the following part of the paper, we use the dimensionless temperature $T \equiv \tilde{T} / \tilde{T}_{a v}$, radius $r \equiv \tilde{r} / \tilde{R}$, time $t \equiv \tilde{t} \tilde{\kappa} / \tilde{R}^{2}$, the angular velocity of the revolution $\omega_{\text {rev }} \equiv \tilde{\omega}_{\text {rev }} \tilde{R}^{2} / \tilde{\kappa}$ and the angular velocity of the rotation $\omega_{\text {rot }} \equiv \tilde{\omega}_{\text {rot }} \tilde{R}^{2} / \tilde{\kappa}$. Note that $T$ is a function of four independent real variables: $(r, \theta, \phi, t)$. The average temperature can be considered to be $T_{a v}=1$. By using these variables, Eq. (1) is expressed as

$$
\frac{\partial T}{\partial t}+\omega_{\text {rot }} \frac{\partial T}{\partial \phi}=\frac{1}{r^{2}}\left\{\frac{\partial}{\partial r}\left(r^{2} \frac{\partial T}{\partial r}\right)+\left[\frac{1}{\sin \theta} \frac{\partial}{\partial \theta}\left(\sin \theta \frac{\partial T}{\partial \theta}\right)+\frac{1}{\sin ^{2} \theta} \frac{\partial^{2} T}{\partial \phi^{2}}\right]\right\} .
$$

The dimensionless form of the boundary condition is given by Eq. (7) of Paper I, and the dimensionless heating rate $\Gamma$ and the global thermal parameter $q_{\text {global }}$ are given by Eqs. (8) and (9), respectively, of Paper I. Note that $q_{\text {global }}$ was denoted by $\lambda$ in Vokrouhlický (1998) and $\chi$ in Vokrouhlický (1999).

\subsection{Iterative solution}

Now, we develop an iterative method for determining a solution of Eq. (3) that satisfies the dimensionless boundary condition given by Eq. (7) of 
Paper I. We choose $T^{(0)}=1$ as the zero-th order solution that satisfies Eq. (3). The $j$-th order solution is expressed as follows:

$$
T^{(j)}=T^{(j-1)}+\delta T^{(j)}
$$

Because Eq. (3) is linear in $T$, its difference $\delta T^{(j)}$ too must satisfy Eq. (3). In order to solve Eq. (3) for $\delta T^{(j)}$, we first expand $\delta T^{(j)}$ by using the Fourier series with respect to time

$$
\delta T^{(j)}(r, \theta, \phi, t)=\sum_{\ell=-\ell_{\max }}^{\ell_{\max }} \delta T_{F, \ell}^{(j)}(r, \theta, \phi) \exp \left(-i \ell \omega_{r e v} t\right),
$$

where $i$ is the imaginary unit, and $\delta T_{F, \ell}^{(j)}(r, \theta, \phi)$ is the complex Fourier coefficient (the subscript "F" shows that they are the Fourier components of the corresponding variable). We should replace $\ell_{\max }$ by $\infty$ for the completeness of the Fourier series. However, we use a finite value of $\ell_{\max }$ for numerical computation. We set $\ell_{\max }=32$ throughout this paper; we have confirmed well convergence using this value for solutions we have obtained. Note that $\delta T_{F, \ell}^{(j)}$ is a function of three independent real variables, $r, \theta, \phi$, and one integer index $\ell$. The Fourier component of $T^{(j)}(r, \theta, \phi, t)$ is expressed as

$$
T_{F, \ell}^{(j)}(r, \theta, \phi)=\delta_{\ell 0}+\sum_{j^{\prime}=1}^{j} \delta T_{F, \ell}^{\left(j^{\prime}\right)}(r, \theta, \phi),
$$

where $\delta_{\ell 0}$ is Kronecker's delta; this term comes from the zero-th order solution $T^{(0)}=1$.

We further expand $\delta T_{F, \ell}^{(j)}(r, \theta, \phi)$ by using the spherical harmonics for $(\theta, \phi)$

$$
\delta T_{F, \ell}^{(j)}(r, \theta, \phi)=\sum_{n=0}^{n_{\max }} \sum_{m=-n}^{n} \delta T_{n, m, \ell}^{(j)}(r) C_{n, m}(\theta, \phi),
$$


where $C_{n, m}(\theta, \phi)$ is a complex spherical harmonics defined just below, and $\delta T_{n, m, \ell}^{(j)}(r)$ is a complex function of a real variable $r$ and three integer indices $(n, m, \ell)$; a method to derive a general solution of $\delta T_{n, m, \ell}^{(j)}(r)$ will be shown below. There are several definitions of the spherical harmonics; we use a definition,

$$
C_{n, m}(\theta, \phi) \equiv(-1)^{(m+|m|) / 2} \sqrt{\frac{(n-|m|) !}{(n+|m|) !}} P_{n}^{|m|}(\cos \theta) \exp (i m \phi),
$$

where $P_{n}^{|m|}(\cos \theta)$ is the associated Legendre function of the first kind. Note that $C_{n, m}=[4 \pi /(2 n+1)]^{1 / 2} Y_{n, m}$, where $Y_{n, m}$ is the normalized spherical harmonics defined, e.g., by Eq. (15.3) of Wigner (1959). Further, note that the spherical harmonics, $C_{n, m}(\theta, \phi)$ with $0 \leq n<\infty$ and $-n \leq m \leq n$ form a complete orthogonal system for a continuous complex function of $(\theta, \phi)$. However, we use a finite value of $n_{\max }$ for numerical computation. We set $n_{\max }=32$ throughout this paper; we confirmed well convergence when we used this value.

Because the thermal conduction equation is linear, we can solve each term in the summation on the RHS of Eq. (7) separately. By substituting Eq. (7) into Eq. (5), and substituting the resultant expression into Eq. (3) and comparing each term proportional to $C_{n, m}(\theta, \phi) \exp \left(-i \ell \omega_{r e v} t\right)$, we get

$$
\begin{aligned}
& \left(-i \ell \omega_{\text {rev }}+i m \omega_{\text {rot }}\right) \delta T_{n, m, \ell}^{(j)}(r) \\
& =\frac{1}{r^{2}}\left\{\frac{d}{d r}\left[r^{2} \frac{d}{d r} \delta T_{n, m, \ell}^{(j)}(r)\right]-n(n+1) \delta T_{n, m, \ell}^{(j)}(r)\right\} .
\end{aligned}
$$

A solution of this equation, that is regular at $r=0$, is $j_{n}\left(\beta_{m, \ell} r\right)$, where $j_{n}$ is the spherical Bessel function of the first kind and $\beta_{m, \ell} \equiv\left(i \ell \omega_{\text {rev }}-i m \omega_{\text {rot }}\right)^{1 / 2}$. 
Among two square roots, we choose

$$
\beta_{m, \ell}= \begin{cases}b_{m, \ell}+i b_{m, \ell} & \text { for } \quad \ell \omega_{r e v} \geq m \omega_{\text {rot }} \\ b_{m, \ell}-i b_{m, \ell} & \text { for } \ell \omega_{r e v}<m \omega_{r o t}\end{cases}
$$

where $b_{m, \ell} \equiv\left(\left|\ell \omega_{\text {rev }}-m \omega_{\text {rot }}\right| / 2\right)^{1 / 2}$. Because $j_{n}\left(\beta_{m, \ell} r\right)$ and its derivative $\beta_{m, \ell} j_{n}^{\prime}\left(\beta_{m, \ell} r\right)$ may take very large or very small values, we multiply them by $B_{n}\left(\beta_{m, \ell}\right)$, a coefficient that depends on $n$ and $\beta_{m, \ell}$, as follows:

$$
\hat{j}_{n}\left(\beta_{m, \ell}, r\right) \equiv B_{n}\left(\beta_{m, \ell}\right) j_{n}\left(\beta_{m, \ell} r\right)
$$

and

$$
\hat{j}_{n}^{\prime}\left(\beta_{m, \ell}, r\right) \equiv B_{n}\left(\beta_{m, \ell}\right) \beta_{m, \ell} j_{n}^{\prime}\left(\beta_{m, \ell} r\right)
$$

in order to get appropriate values for computation. Note that $\hat{j}_{n}\left(\beta_{m, \ell}, r\right)$ and $\hat{j}_{n}^{\prime}\left(\beta_{m, \ell}, r\right)$ are functions of a complex number $\beta_{m, \ell}$, a real number $r$ and an integer index $n$. We find it convenient to use the following equations for determining $B_{n}\left(\beta_{m, \ell}\right)$ :

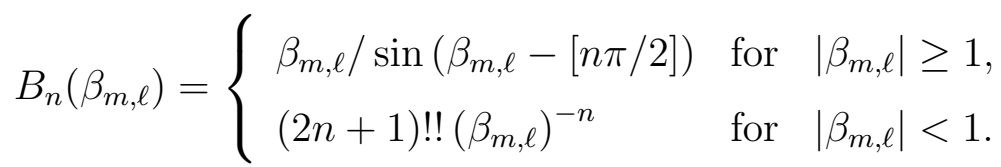

Note that we get $\hat{j}_{n}\left(\beta_{m, \ell}, r\right)=r^{n}$ and $\hat{j}_{n}^{\prime}\left(\beta_{m, \ell}, r\right)=n r^{n-1}$ if $\beta_{m, \ell}=0$ (i.e., $\left.\ell \omega_{\text {rev }}=m \omega_{\text {rot }}\right)$. The detailed procedures for calculating $\hat{j}_{n}\left(\beta_{m, \ell}, r\right)$ and $\hat{j}_{n}^{\prime}\left(\beta_{m, \ell}, r\right)$ using $B_{n}\left(\beta_{m, \ell}\right)$ determined by Eq. (13) are given in Appendix A of Paper I, although we did not realize that $\hat{j}$ and $\hat{j}^{\prime}$ are determined by a set of three variables $\beta_{m}, r$ and $n$ when we wrote Paper I, and we used inappropriate expressions $\hat{j}_{n, m}\left(\beta_{m}\right)$ and $\hat{j}_{n, m}^{\prime}\left(\beta_{m}\right)$, that should be replaced by $\hat{j}_{n}\left(\beta_{m}, 1\right)$ and $\hat{j}_{n}^{\prime}\left(\beta_{m}, 1\right)$. 
Hence, we obtain a general solution of Eq. (9) that is regular at $r=0$

$$
\delta T_{n, m, \ell}^{(j)}(r)=\delta \mathcal{T}_{n, m, \ell}^{(j)} \hat{j}_{n}\left(\beta_{m, \ell}, r\right)
$$

where $\delta \mathcal{T}_{n, m, \ell}^{(j)}$ is a constant complex number, and it will be determined on the basis of the surface boundary condition. Substituting Eq. (14) into Eq. (7), and substituting the result of which, in turn, into Eq. (5), we get a general solution of Eq. (3):

$\delta T^{(j)}(r, \theta, \phi, t)=\sum_{\ell=-\ell_{\max }}^{\ell_{\max }} \sum_{n=0}^{n_{\max }} \sum_{m=-n}^{n} \delta \mathcal{T}_{n, m, \ell}^{(j)} \hat{j}_{n}\left(\beta_{m, \ell}, r\right) C_{n, m}(\theta, \phi) \exp \left(-i \ell \omega_{\text {rev }} t\right)$.

Substituting Eq. (4) into the surface boundary condition given by Eq.

(7) of Paper I, we get

$$
\left[\frac{1}{4}\left(T^{(j-1)}+\delta T^{(j)}\right)^{4}+q_{\text {global }}\left(\frac{\partial T^{(j-1)}}{\partial r}+\frac{\partial \delta T^{(j)}}{\partial r}\right)\right]_{r=1}=\Gamma(\cos \Theta) .
$$

We expect that $\delta T^{(j)}$ is on the order of $o\left(\varepsilon^{j}\right)$, where $\varepsilon$ is a positive number less than unity. By expanding the nonlinear term using $\delta T^{(j)}$ and omitting the terms on the order of $o\left(\varepsilon^{j}\right)$, we get $\left(T^{(j-1)}+\delta T^{(j)}\right)^{4} \approx\left(T^{(j-1)}\right)^{4}+4 \delta T^{(j)}$. Hence, we get the following boundary condition:

$$
\left[\delta T^{(j)}+q_{\text {global }} \frac{\partial \delta T^{(j)}}{\partial r}\right]_{r=1}=\Gamma(\cos \Theta)-\Lambda^{(j-1)}(\theta, \phi, t)-q_{\text {global }}\left[\frac{\partial T^{(j-1)}}{\partial r}\right]_{r=1},
$$

where we denote the dimensionless radiative-cooling-rate by $\Lambda^{(j-1)}(\theta, \phi, t) \equiv$ $\left[\frac{1}{4}\left(T^{(j-1)}\right)^{4}\right]_{r=1}$.

Because we have already obtained the general solutions of the thermal conduction equation for $\delta T^{(j)}$ by performing the expansion on the basis of 
the spherical harmonics and the Fourier series, and because Eq. (17) is linear in $\delta T^{(j)}$, we can get a solution that satisfies Eq. (17) by expanding the righthand side (RHS) of Eq. (17) on the basis of the spherical harmonics and the Fourier series. The last term on the RHS of Eq. (17) has been already determined when we are going to calculate $\delta T^{(j)}$ by employing the iterative method.

\section{Figure 3}

The first term on the RHS of Eq. (17) is expressed as follows:

$$
\Gamma(\cos \Theta)=\sum_{n=0}^{n_{\max }} \Gamma_{n} P_{n}(\cos \Theta),
$$

(see Eq. (25)-(27) of Paper I as for the values of the coefficients $\Gamma_{n}$ ). In order to calculate $P_{n}(\cos \Theta)$, we use a different coordinate system $\left(\tilde{x}^{\prime}, \tilde{y}, \tilde{z}^{\prime}\right)$ (see Fig. 3); the origin and $\tilde{y}$ axis of this coordinate system are identical to those of the coordinate system $(\tilde{x}, \tilde{y}, \tilde{z}) ; \tilde{z}^{\prime}$ axis is set in the direction of the orbital angular momentum of the body in the inertial frame whose origin is at the star; $\tilde{x}^{\prime}$ axis is set so that $\tilde{x}^{\prime}, \tilde{y}$ and $\tilde{z}^{\prime}$ form a right-handed coordinate system. The coordinate system $\left(\tilde{x}^{\prime}, \tilde{y}, \tilde{z}^{\prime}\right)$ does not rotate with respect to the inertial frame. The unit vector in the direction of the star viewed from the center of the body is expressed as $\boldsymbol{n}_{*}=\left(\cos \left(\omega_{\text {rev }} t\right), \sin \left(\omega_{\text {rev }} t\right), 0\right)$ in coordinate system $\left(\tilde{x}^{\prime}, \tilde{y}, \tilde{z}^{\prime}\right)$, where we set $t=0$ at an instant of the summer solstice. We define a corresponding spherical coordinates $\left(\tilde{r}, \theta^{\prime}, \phi^{\prime}\right)$ in the usual manner as shown in Fig. 3. Because $\Theta$ is the angle between two points with sets of the colatitude and the longitude $\left(\theta^{\prime}, \phi^{\prime}\right)$ and $\left(\pi / 2, \omega_{\text {rev }} t\right)$ when viewed from the center of the body as drawn in Fig. 3, the Legendre polynomial as a function 
of $\cos \Theta$ is expressed using these variables according to the addition theorem (e.g., Arfken , 1970, Eq. (12.200))

$$
\begin{aligned}
P_{n}(\cos \Theta) & =\sum_{\ell=-n}^{n} C_{n, \ell}\left(\theta^{\prime}, \phi^{\prime}\right) C_{n, \ell}^{*}\left(\pi / 2, \omega_{r e v} t\right) \\
& =\sum_{\ell=-n}^{n} C_{n, \ell}(\pi / 2,0) C_{n, \ell}\left(\theta^{\prime}, \phi^{\prime}\right) \exp \left(-i \ell \omega_{r e v} t\right) .
\end{aligned}
$$

Because $(\tilde{x}, \tilde{y}, \tilde{z})$ system is given by rotating $\left(\tilde{x}^{\prime}, \tilde{y}, \tilde{z}^{\prime}\right)$ system with respect to the $\tilde{y}$ axis at an angle $\gamma$, we get

$$
C_{n, \ell}\left(\theta^{\prime}, \phi^{\prime}\right)=\sum_{m=-n}^{n} C_{n, m}(\theta, \phi) \mathcal{D}^{(n)}(\{0, \gamma, 0\})_{m, \ell}
$$

from Eq. (15.5) of Wigner (1959), where the elements of the transformation coefficient are given by Eq. (15.27) of Wigner (1959):

$$
\begin{array}{r}
\mathcal{D}^{(n)}(\{0, \gamma, 0\})_{m, \ell}=\sum_{k}(-1)^{k} \frac{[(n+\ell) !(n-\ell) !(n+m) !(n-m) !]^{1 / 2}}{(n-m-k) !(n+\ell-k) ! k !(k+m-\ell) !} \\
\times \cos ^{2 n+\ell-m-2 k}(\gamma / 2) \sin ^{2 k+m-\ell}(\gamma / 2),
\end{array}
$$

where integer $k$ varies within the range so that all arguments in factorials are greater than or equal to zero. Hence, we get complete expansion of the heating term by using the spherical harmonics as well as the Fourier series:

$$
\Gamma(\cos \Theta)=\sum_{n=0}^{n_{\max }} \sum_{m=-n}^{n} \sum_{\ell=-n}^{n} \Gamma_{n, m, \ell} C_{n, m}(\theta, \phi) \exp \left(-i \ell \omega_{\text {rev }} t\right),
$$

where

$$
\Gamma_{n, m, \ell}=\Gamma_{n} C_{n, \ell}(\pi / 2,0) \mathcal{D}^{(n)}(\{0, \gamma, 0\})_{m, \ell} .
$$

Next, we express the second term on the RHS of Eq. (17) by using the Fourier series:

$$
\Lambda^{(j)}(\theta, \phi, t)=\frac{1}{4}\left[T^{(j)}(1, \theta, \phi, t)\right]^{4}=\sum_{\ell=-\ell_{\max }}^{\ell_{\max }} \Lambda_{F, \ell}^{(j)}(\theta, \phi) \exp \left(-i \ell \omega_{r e v} t\right),
$$


where the first argument " 1 " of $T^{(j)}$ shows that it is the temperature at the surface of the body where $r=1$. Note that $\Lambda^{(j)}(\theta, \phi, t)$ is a real number, but its Fourier component $\Lambda_{F, \ell}^{(j)}(\theta, \phi)$ is a complex number. Further, note that we used superscript $(j)$ in the above equations to represent a general relation, but we should replace $(j)$ by $(j-1)$ when we apply this relation to Eq. (17). The Fourier component is given by

$$
\begin{aligned}
& \Lambda_{F, \ell}^{(j)}(\theta, \phi) \\
& =\frac{1}{4}\left[\sum_{\ell_{1}+\ell_{2}+\ell_{3}+\ell_{4}=\ell} T_{F, \ell_{1}}^{(j)}(1, \theta, \phi) T_{F, \ell_{2}}^{(j)}(1, \theta, \phi) T_{F, \ell_{3}}^{(j)}(1, \theta, \phi) T_{F, \ell_{4}}^{(j)}(1, \theta, \phi)\right],
\end{aligned}
$$

where the summation is performed for all repeated permutation of four integers $-\ell_{\max } \leq \ell_{p} \leq \ell_{\max }(p=1,2,3,4)$ with the condition that the sum of the four integers should be equal to a given value of $\ell$.

We further expand $\Lambda_{F, \ell}^{(j)}(\theta, \phi)$ by using the spherical harmonics:

$$
\Lambda_{F, \ell}^{(j)}(\theta, \phi)=\sum_{n=0}^{n_{\max }} \sum_{m=-n}^{n} \Lambda_{n, m, \ell}^{(j)} C_{n, m}(\theta, \phi),
$$

where $\Lambda_{n, m, \ell}^{(j)}$ is a constant complex number, the value of which is calculated by using the orthogonal relation of the spherical harmonics:

$$
\int_{0}^{\pi} d \theta \sin \theta \int_{-\pi}^{\pi} d \phi C_{n, m}(\theta, \phi) C_{n^{\prime}, m^{\prime}}^{*}(\theta, \phi)=\frac{4 \pi}{2 n+1} \delta_{n, n^{\prime}} \delta_{m, m^{\prime}},
$$

where $\delta_{n, n^{\prime}}$ and $\delta_{m, m^{\prime}}$ are Kronecker's deltas. Hence, we get

$$
\Lambda_{n, m, \ell}^{(j)}=\frac{2 n+1}{4 \pi} \int_{0}^{\pi} d \theta \sin \theta \int_{-\pi}^{\pi} d \phi \Lambda_{F, \ell}^{(j)}(\theta, \phi) C_{n, m}^{*}(\theta, \phi) .
$$

This double integral is numerically calculated by using the quadrature by parts; we used 360 and 720 meshes for ranges $0 \leq \theta \leq \pi$ and $-\pi \leq \phi \leq \pi$, respectively, in the integration. 
Thus, by using the spherical harmonics with respect to $(\theta, \phi)$ and the Fourier series with respect to $t$, we can expand all the three terms on the RHS of Eq. (17). By comparing terms with each set of $(n, m, \ell)$, we get

$$
\delta \mathcal{T}_{n, m, \ell}^{(j)}=f_{\text {relax }} \frac{\Gamma_{n, m, \ell}-\Lambda_{n, m, \ell}^{(j-1)}-q_{\text {global }} \mathcal{T}_{n, m, \ell}^{(j-1)} \hat{j}_{n}^{\prime}\left(\beta_{m, \ell}, 1\right)}{\hat{j}_{n}\left(\beta_{m, \ell}, 1\right)+q_{\text {global }} \hat{j}_{n}^{\prime}\left(\beta_{m, \ell}, 1\right)},
$$

where $\mathcal{T}_{n, m, \ell}^{(j-1)}=\delta_{n, 0} \delta_{m, 0} \delta_{\ell, 0}+\sum_{k=1}^{j-1} \delta \mathcal{T}_{n, m, \ell}^{(k)}$. The factor $f_{\text {relax }}$ should be equal to unity in order to satisfy Eq. (17). If $\delta T^{(j)}$ that satisfies Eq. (17) is on the order of $\varepsilon^{j}$ with $0 \leq \varepsilon<1$, the summation $\sum_{0}^{\infty} \delta T^{(j)}$ would converges. However, we found that the summation does not converge for parameters we used. Hence, we set $f_{\text {relax }}=0.5$ throughout this paper for obtaining iterative solutions; the summation always converged by using $f_{\text {relax }}=0.5$. On the other hand, the linear solution is obtained by setting $f_{\text {relax }}=1$ and $j=1$ in Eq. (29).

We define the RMS error of the boundary condition for Fourier component $\ell$ by

$$
\begin{aligned}
\Delta_{\ell}^{(j)} \equiv & \left\{\frac{1}{4 \pi} \int_{-1}^{1} d \cos \theta \int_{-\pi}^{\pi} d \phi\right. \\
& \left.\left|\Gamma_{F, \ell}(\theta, \phi)-\Lambda_{F, \ell}^{(j)}(\theta, \phi)-q_{g l o b a l}\left[\frac{\partial T_{F, \ell}^{(j)}(r, \theta, \phi)}{\partial r}\right]_{r=1}\right|^{2}\right\}^{1 / 2},(30
\end{aligned}
$$

where the Fourier transform of the heating term is given by

$$
\Gamma_{F, \ell}(\theta, \phi)=\sum_{n=0}^{n_{\max }} \sum_{m=-n}^{n} \Gamma_{n, m, \ell} C_{n, m}(\theta, \phi) .
$$

We continued the iteration until $\Delta_{\ell} \leq 1 \times 10^{-3}$ was satisfied for all the Fourier components $\left(-\ell_{\max } \leq \ell \leq \ell_{\max }\right)$. In principle, we can obtain a solution that satisfies the nonlinear boundary condition with an arbitrary accuracy by using large values of $\ell_{\max }$ and $n_{\max }$, and a large number of meshes 
for performing numerical integration of Eq. (28), and repeatedly using the above-mentioned recurrence formulas as many times as needed.

\subsection{Calculation of the thermal force}

The force that acts on a spherical body due to the thermal emission for the temperature distribution determined by $j$-th iteration is given by

$$
\begin{aligned}
& \tilde{\boldsymbol{f}}^{(j)}=-\tilde{R}^{2} \int_{-\pi}^{\pi} d \phi \int_{0}^{\pi} d \theta \sin \theta\left\{2 \epsilon \tilde{\sigma}\left[\tilde{T}^{(j)}(\tilde{R}, \theta, \phi)\right]^{4} /(3 \tilde{c})\right\} \hat{\boldsymbol{n}} \\
= & -\frac{2 \tilde{R}^{2} \alpha \tilde{F}_{*}}{3 \tilde{c}} \int_{-\pi}^{\pi} d \phi \int_{0}^{\pi} d \theta \sin \theta \Lambda^{(j)}(\theta, \phi, t)(\sin \theta \cos \phi \hat{\boldsymbol{x}}+\sin \theta \sin \phi \hat{\boldsymbol{y}}+\cos \theta \hat{\boldsymbol{z}}),
\end{aligned}
$$

where $\tilde{c}$ is the speed of light, $\hat{\boldsymbol{n}}$ is the unit vector normal to the surface of the spherical body, and $\hat{\boldsymbol{x}}, \hat{\boldsymbol{y}}$ and $\hat{\boldsymbol{z}}$ are the unit vectors in the directions of $\tilde{x}^{-}$, $\tilde{y}$ - and $\tilde{z}$-axes, respectively. By expanding $\Lambda^{(j)}(\theta, \phi)$ in Eq. (32) using Eqs. (24) and (26), and using orthogonal relation given by Eq. (27), we get

$$
\tilde{f}_{x}^{(j)}-i \tilde{f}_{y}^{(j)}=\frac{8 \sqrt{2} \pi \tilde{R}^{2} \alpha \tilde{F}_{*}}{9 \tilde{c}} \sum_{\ell=-\ell_{\max }}^{\ell_{\max }} \Lambda_{1,1, \ell}^{(j)} \exp \left(-i \ell \omega_{\text {rev }} t\right)
$$

and

$$
\tilde{f}_{z}^{(j)}=-\frac{8 \pi \tilde{R}^{2} \alpha \tilde{F}_{*}}{9 \tilde{c}} \sum_{\ell=-\ell_{\max }}^{\ell_{\max }} \Lambda_{1,0, \ell}^{(j)} \exp \left(-i \ell \omega_{\text {rev }} t\right),
$$

where $\tilde{\boldsymbol{f}}^{(j)}=\tilde{f}_{x}^{(j)} \hat{\boldsymbol{x}}+\tilde{f}_{y}^{(j)} \hat{\boldsymbol{y}}+\tilde{f}_{z}^{(j)} \hat{\boldsymbol{z}}$. The unit vector in the direction of revolution velocity is expressed as $\hat{\boldsymbol{v}}=\cos \gamma \sin \left(\omega_{\text {rev }} t\right) \hat{\boldsymbol{x}}-\cos \left(\omega_{\text {rev }} t\right) \hat{\boldsymbol{y}}+$ $\sin \gamma \sin \left(\omega_{\text {rev }} t\right) \hat{\boldsymbol{z}}$. The time-averaged rate of change in the semimajor axis is given by

$$
\left\langle\dot{\tilde{a}}^{(j)}\right\rangle_{t}=2<\tilde{\boldsymbol{f}}^{(j)} \cdot \hat{\boldsymbol{v}}>_{t} /\left(\tilde{m} \tilde{\omega}_{r e v}\right),
$$


where $\tilde{m}$ is the mass of the body. We define the dimensionless rate of change in the semimajor axis, $\left\langle\dot{a}^{(j)}\right\rangle_{t} \equiv\left\langle\dot{\tilde{a}}^{(j)}\right\rangle_{t} \tilde{\omega}_{r e v} / \tilde{\Phi}$, where $\tilde{\Phi}=\pi \tilde{R}^{2} \alpha \tilde{F}_{*} /(\tilde{m} \tilde{c})$ is the usual radiation force factor. The dimensionless rate $\left\langle\dot{a}^{(j)}\right\rangle_{t}$ is expressed as the sum of two terms:

$$
\left\langle\dot{a}_{s}^{(j)}\right\rangle_{t}=\frac{8}{9} \sin \gamma\left\{-\Im\left[\Lambda_{1,0,1}^{(j)}\right]+\Im\left[\Lambda_{1,0,-1}^{(j)}\right]\right\}
$$

and

$$
\left\langle\dot{a}_{d}^{(j)}\right\rangle_{t}=\frac{8 \sqrt{2}}{9}\left\{(1+\cos \gamma) \Im\left[\Lambda_{1,1,1}^{(j)}\right]+(1-\cos \gamma) \Im\left[\Lambda_{1,1,-1}^{(j)}\right]\right\},
$$

where $\Im$ represents the imaginary part of a complex number. These two parts correspond to the "seasonal component" and the "diurnal/mixed components" of the rate of change in the semimajor axis based on the linear solution obtained by Vokrouhlický (1999). The seasonal component, $\left\langle\dot{a}_{s}^{(j)}\right\rangle_{t}$, is strictly equal to zero, when $\gamma=0^{\circ}$ and $180^{\circ}$, as seen from Eq. (36). On the other hand, the diurnal/mixed components $\left\langle\dot{a}_{d}^{(j)}\right\rangle_{t}$ is not necessarily equal to zero, even if $\gamma=90^{\circ}$ as was found for the linear solutions by Vokrouhlický (1999).

The dimensionless rate of change of the semimajor axis obtained by using the linear approximation, $<\dot{a}>_{t, \text { linear }}$, is expressed as the sum of the following two terms (Vokrouhlický, 1999):

$$
<\dot{a}_{s}>_{t, \text { linear }}=\frac{8}{9}\left[-\frac{\sin ^{2} \gamma}{4}\left(\mathcal{E}_{1,0,1}-\mathcal{E}_{1,0,-1}\right)\right]
$$

and

$$
<\dot{a}_{d}>_{t, \text { linear }}=\frac{8}{9}\left[-\cos ^{4}(\gamma / 2) \mathcal{E}_{1,1,1}+\sin ^{4}(\gamma / 2) \mathcal{E}_{1,1,-1}\right]
$$

where

$$
\mathcal{E}_{n, m, \ell}=\Im\left[\frac{1}{1+q_{\text {global }}\left\{\hat{j}_{n}^{\prime}\left(\beta_{m, \ell}, 1\right) / \hat{j}_{n}\left(\beta_{m, \ell}, 1\right)\right\}}\right]
$$




\subsection{Dimensionless parameters}

The dimensionless temperature distribution (i.e., the dimensional temperature distribution normalized by the average temperature) as a function of $\theta, \phi$ and $t$ is determined on the basis of four dimensionless parameters: $q_{\text {global }}, \omega_{\text {rot }}$ and $\omega_{\text {rev }}$, and $\gamma$. The global thermal parameter defined by Eq. (9) of Paper I is expressed as $q_{g l o b a l}=\tilde{R}_{c} / \tilde{R}$ where $\tilde{R}_{c}$ is the critical radius defined by Eq. (37) of Paper I. The thermal parameters of the rotation and the revolution are defined as

$$
q_{\text {rot }} \equiv \frac{\tilde{k}_{T} \tilde{T}_{a v} / \tilde{l}_{\text {rot }}}{\alpha \tilde{F}_{*}}=\omega_{\text {rot }}^{1 / 2} q_{\text {global }}=\left(\tilde{P}_{c} / \tilde{P}_{\text {rot }}\right)^{1 / 2},
$$

and

$$
q_{\text {rev }} \equiv \frac{\tilde{k}_{T} \tilde{T}_{a v} / \tilde{l}_{\text {rev }}}{\alpha \tilde{F}_{*}}=\omega_{\text {rev }}^{1 / 2} q_{\text {global }}=\left(\tilde{P}_{c} / \tilde{P}_{\text {rev }}\right)^{1 / 2}
$$

respectively. Here, $\tilde{l}_{\text {rot }} \equiv\left(\tilde{\kappa} / \tilde{\omega}_{\text {rot }}\right)^{1 / 2}$ and $\tilde{l}_{\text {rev }} \equiv\left(\tilde{\kappa} / \tilde{\omega}_{\text {rev }}\right)^{1 / 2}$ are penetration depths of thermal waves for rotation and revolution, respectively, and the critical period $\tilde{P}_{c}$ is given by Eq. (38) of Paper I. Note that the values of $\omega_{\text {rot }}$ and $\omega_{\text {rev }}$ are determined from Eqs. (41) and (42) if the values of $q_{\text {global }}, q_{\text {rot }}$ and $q_{\text {rev }}$ are given.

\section{Results and Discussion}

We here show temperature distributions on the surface of a spherical asteroid that has $100 \mathrm{~m}$ in the physical radius, and $2.6 \mathrm{AU}$ in the orbital radius as an example. We assume that the asteroid has the representative values of the thermal conduction coefficient and the thermometric conductivity of ordinary chondrites, $\tilde{k}_{T}=1 \mathrm{~W} \mathrm{~m}^{-1} \mathrm{~K}^{-1}$ and $\tilde{\kappa}=1 \times 10^{-6} \mathrm{~m}^{2} \mathrm{~s}^{-1}$, respectively (Yomogida and Matsui, 1983; Opeil et al., 2010); we further assume that 
$\alpha=1$ and $\epsilon=1$ for simplicity. In this case, we get $q_{\text {rev }} \approx 0.2$ and $q_{\text {global }} \approx$ 0.01. We employ the relation of the rotation period and the physical radius determined by Kadono et al. (2009), $\tilde{P}_{\text {rot }}(\mathrm{s}) \approx 20 \tilde{R}(\mathrm{~m})$; thus, we get $q_{\text {rot }} \approx 50$. The temperature distributions are calculated at the summer solstice and the autumn equinox, i.e. $\omega_{\text {rev }} t=0^{\circ}$ and $90^{\circ}$, respectively, in the case of $\gamma=60^{\circ}$, as an example.

\section{Figure 4}

Fig. 4 (a) and (b) shows the Mollweide projections of the temperature distributions at the summer solstice $\left(\omega_{\text {rev }} t=0^{\circ}\right)$ determined by (a) the iterative method $\left(f_{\text {relax }}=0.5\right.$ and $\left.j=16\right)$ and (b) the linear approximation $\left(f_{\text {relax }}=1\right.$ and $\left.j=1\right)$, respectively. The substellar point is shown by a symbol " $\star$ ", the coordinates of which is $(\theta, \phi)=\left(30^{\circ}, 0^{\circ}\right)$ at the summer solstice (see Figs. 1 and 2 as for the definition of the coordinates). The eastern and western ends on the equator in the Mollweide projections denoted by "E" and "W", respectively, show an identical point where it is the midnight with coordinates $(\theta, \phi)=\left(90^{\circ}, 180^{\circ}\right)$ and $(\theta, \phi)=\left(90^{\circ},-180^{\circ}\right)$ at the summer solstice. The temperature is almost constant for the longitude at an each colatitude in both the iterative and linear solutions. This is because the temperature is averaged out over whole range of the longitude because of fast rotation in the case of $q_{r o t}=50$, i.e. $\tilde{P}_{r o t}=4 \times 10^{-4} \tilde{P}_{c}$. The maximum dimensionless-temperature is achieved around the north pole $(T=1.34$ for the iterative solution and $T=1.53$ for the linear solution). It is rather difficult to determine an accurate position at which the temperature has the maximum value, because the temperature distribution is very flat around the north pole. The minimum dimensionless-temperature is achieved at the 
south pole in both the iterative solution $(T=0.64)$ and the linear solution $(T=0.78)$. Both the maximum and minimum temperatures of the iterative solution are lower than those for the linear solution. This is because the dimensionless form of the cooling rate $T^{4} / 4=(1+\delta T)^{4} / 4$ used to determine the nonlinear solution is larger than $(1 / 4)+\delta T$ used to determine the linear solution for a certain value of $\delta T$.

\section{Figure 5}

Fig. 5 shows the temperature distributions of (a) iterative and (b) linear solutions at the autumn equinox, $\omega_{\text {rev }} t=90^{\circ}$, with the same parameters as Fig. 4. The substellar point at the autumn equinox with coordinates $(\theta, \phi)=\left(90^{\circ}, 90^{\circ}\right)$ locates at the center of the Mollweide projections. Note that we use a coordinate system that keeps a same direction with respect to the inertial frame as shown in Figs. 1 and 2; therefore the sunup and the sunset occur at $\phi=0^{\circ}$ and $180^{\circ}$, respectively, at the autumn equinox. The eastern and western ends on the equator in the Mollweide projections denoted by "E" and "W", respectively, show an identical point where it is the midnight on the equator with coordinates $(\theta, \phi)=\left(90^{\circ}, 270^{\circ}\right)$ and $(\theta, \phi)=\left(90^{\circ},-90^{\circ}\right)$, at the autumn equinox. The temperature dependence on the colatitude of the iterative solution shown in Fig. 5 (a) is different from that of the linear solution shown in Fig. 5 (b): It is seen in Fig. 5 (a) that the south pole is very cold $(T=0.60)$ for the iterative solution, because the south pole has been in the polar night until the autumn equinox. In contrast, Fig. 5(b) shows that the south pole is not so cold $(T=0.77)$. This is again attributed to the difference in the nonlinear and linear cooling rates. On the other hand, both the iterative and linear temperature distributions 
show the same value of the maximum temperature, $T=1.07$, at almost same positions, $(\theta, \phi)=\left(82^{\circ}, 126^{\circ}\right)$ for the iterative solution and $\left(81^{\circ}, 126^{\circ}\right)$ for the linear solution. This is because $\delta T=0.07$ is sufficiently less than unity so that the linear approximation is good.

For comparison, we also obtained the iterative and linear solutions for the thermal conduction coefficient $\tilde{k}_{T}=1 \times 10^{-3} \mathrm{~W} \mathrm{~m}^{-1} \mathrm{~K}^{-1}$ and the thermometric conductivity $\tilde{\kappa}=1 \times 10^{-9} \mathrm{~m}^{2} \mathrm{~s}^{-1}$; these values are a thousandth of those of ordinary chondrites. Such low values may be realized for an asteroid covered by a thick regolith layer (Bottke et al., 2006). Although it is more precise to employ a model developed by Vokrouhlický and Brož (1999) in which a regolith shell with a low thermal conductivity covers a remaining rocky body with a high thermal conductivity, we here use our model with the uniform thermal and thermometric conductivities assuming that the regolith is thicker than the penetrating depth of the seasonal thermal wave. The values of dimensionless parameters are estimated to be $q_{\text {global }}=1 \times 10^{-5}$, $q_{\text {rot }}=1.5$ and $q_{\text {rev }}=6 \times 10^{-3}$.

\section{Figure 6}

Fig. 6 shows the Mollweide projections of the temperature distributions at the summer solstice for $q_{\text {global }}=1 \times 10^{-5}, q_{\text {rot }}=1.5, q_{\text {rev }}=6 \times 10^{-3}$ and $\gamma=60^{\circ}$ determined by (a) the iterative method $\left(f_{\text {relax }}=0.5\right.$ and $\left.j=41\right)$ and (b) the linear approximation $\left(f_{\text {relax }}=1\right.$ and $\left.j=1\right)$. The minimum dimensionless-temperature is achieved at the south pole in both the iterative solution $(T=0.40)$ and the linear solution $(T=0.75)$. The value of the minimum dimensionless-temperature for the iterative solution $(T=0.40)$ is 
much less than that in Fig. 4(a) $(T=0.64)$, because the seasonal thermal inertia is effective in the case of Fig. 4 for $q_{\text {rev }}=0.2$, but is ineffective in the case of Fig. 6 for $q_{r e v}=6 \times 10^{-3}$, and the south pole cools down rapidly during the polar night. The difference in the minimum temperatures is not clearly seen in the linear solutions: $T=0.78$ in Fig. $4(\mathrm{~b})$, and $T=0.75$ in Fig. 6(b). The maximum values of the dimensionless-temperature are $T=1.39$ at $(\theta, \phi)=\left(22^{\circ}, 15^{\circ}\right)$ for the iterative solution, and $T=1.64$ at $(\theta, \phi)=\left(14^{\circ}, 27^{\circ}\right)$ for the linear solution. Note that it is rather difficult to determine an accurate position at which the temperature has the maximum value, because the temperature distribution is very flat around its maximum. Both the maximum and minimum temperatures of the iterative solution are lower than those for the linear solution as in Fig. 4 for the same reason. A major difference in Fig. 6 compared to Fig. 4 is that the diurnal variation of the temperature is clearly seen for a constant colatitude. For example, at the equator with $\theta=90^{\circ}$, the maximum temperature $(T=1.03)$ is achieved at $\phi=24^{\circ}$ in the afternoon (note that $\phi=0^{\circ}$ at noon), and the minimum temperature $(T=0.77)$ is achieved at $\phi=-92^{\circ}$ just before the sunrise $\left(\phi=-90^{\circ}\right)$ for the iterative solution, and similar variation is seen also in the linear solution.

\section{Figure 7}

The temperature distributions in Fig. 7 are also different from those in Fig. 5; the diurnal variation of the temperature is large for both the iterative and linear solutions. The maximum temperature is $T=1.28$ at $(\theta, \phi)=$ $\left(90^{\circ}, 109^{\circ}\right)$ for the iterative solution, and $T=1.35$ at $(\theta, \phi)=\left(90^{\circ}, 113^{\circ}\right)$ for the linear solution. By noting that the coordinates of the substellar point at 
the autumn equinox are $(\theta, \phi)=\left(90^{\circ}, 90^{\circ}\right)$, the angle between the maximum temperature point and the substellar point is estimated to be about $20^{\circ}$ for both the iterative and linear solutions; the maximum temperature is attained about $0.06 P_{\text {rot }}$ after the noon when viewed on a fixed point on the equator of the body. A large diurnal variation of the temperature accompanied with a significant angle of the maximum-temperature point and the substellar point when viewed from the center of a body is characteristic to the case when $q_{\text {rot }} \sim 1$ with $q_{\text {global }} \lesssim 1$ (see Fig. 5 of Paper I, in which we used the notation $q_{\text {thermal }}$ instead of $q_{\text {rot }}$ ). It is also seen that the north and south poles are very cold ( $T=0.53$ for the north pole and $T=0.47$ for the south pole) in the case of the iterative solution; because $q_{\text {rev }} \ll 1$, the seasonal thermal inertia is ineffective and the north pole cools down to a temperature similar to that of the south pole in a timescale much shorter than the revolution period, even though the midnight sun continued to irradiate the north pole until the autumn equinox. The temperatures at both poles in the case of the linear solution is $T=0.76$; this value is very different from those of iterative solution. The maximum and minimum temperatures of the linear solutions are larger than those of iterative solutions. This is again attributed to the smaller cooling rate used in the linear approximation compared to the precise cooling rate used in the iterative method.

The dimensionless rate of change in the semimajor axis is listed in Table 1 for two sets of values of $\left(q_{\text {global }}, q_{\text {rot }}, q_{\text {rev }}\right)$ with various values of $\gamma$; the former set is that corresponds to Figs. 4 and 5, and the latter to Figs. 6 and 7. The value of $\left\langle\dot{a}_{s}^{(j)}\right\rangle_{t}$ is strictly equal to zero when $\gamma=0^{\circ}$ and $180^{\circ}$, and otherwise it is negative; the absolute value of $\left\langle\dot{a}_{s}^{(j)}\right\rangle_{t}$ is maximum at $\gamma=90^{\circ}$. The 
absolute value of $\left\langle\dot{a}_{d}^{(j)}\right\rangle_{t}$ have maximum value when $\gamma=0^{\circ}$ and $180^{\circ}$. The value of $\left\langle\dot{a}_{d}^{(j)}\right\rangle_{t}$ is not completely equal to zero when $\gamma=90^{\circ}$ because of the diurnal-seasonal mixed effect (Vokrouhlický, 1999); however, it is negligibly small, similarly to the case of the linear solution obtained by Vokrouhlický (1999). Note that the absolute values of $\left\langle\dot{a}^{(j)}\right\rangle_{t}$ has a very small value for $q_{\text {global }}=1 \times 10^{-2}, q_{\text {rev }}=0.2, q_{\text {rot }}=50$ and $\gamma=30^{\circ}$, because $\left\langle\dot{a}_{d}^{(j)}\right\rangle_{t}$ and $\left\langle\dot{a}_{s}^{(j)}\right\rangle_{t}$ cancel each other. Similar cancelation is seen in the linear solution for the same parameter set. On the other hand, the small absolute value of $\left\langle\dot{a}^{(j)}\right\rangle_{t}$ for $q_{\text {global }}=1 \times 10^{-5}, q_{\text {rev }}=6 \times 10^{-3}, q_{\text {rot }}=1.5$ and $\gamma=90^{\circ}$ is attributed to the small value of $q_{\text {rev }}$ for which the value of $\left\langle\dot{a}_{s}^{(j)}\right\rangle_{t}$ is small (Note that $\left\langle\dot{a}_{d}^{(j)}\right\rangle_{t}$ is very small for $\gamma=90^{\circ}$ as described above).

In order to examine the difference between $\left\langle\dot{a}^{(j)}\right\rangle_{t}$ and $\left\langle\dot{a}_{\text {linear }}^{(j)}\right\rangle_{t}$, we calculated the values of them for wide range of dimensionless parameters corresponding to $1 \times 10^{-3} \leq \tilde{k}_{T}\left[\mathrm{~W} \mathrm{~m}^{-1} \mathrm{~K}^{-1}\right] \leq 1$ and $1 \leq \tilde{R}[\mathrm{~m}] \leq 10^{3}$ at 2.6AU from the sun with $\gamma=0^{\circ}$ and $90^{\circ}$ as shown in Table 2. Because the values of parameter set $\left(q_{\text {global }}, q_{\text {rev }}, q_{\text {rot }}, \gamma\right)$ are given for the calculation of the change rates of semimajor axis in Table 2, these values of parameter set are accurate; on the other hand, the values of $\tilde{k}_{T}$ and $\tilde{R}$ are written to only one significant figure. As described above, $\left\langle\dot{a}^{(j)}\right\rangle_{t}=\left\langle\dot{a}_{d}^{(j)}\right\rangle_{t}$ for $\gamma=0^{\circ}$, and $\left\langle\dot{a}^{(j)}\right\rangle_{t} \approx\left\langle\dot{a}_{s}^{(j)}\right\rangle_{t}$ for $\gamma=90^{\circ}$. We have not calculated the rate of change of the semimajor axis for other values of $\gamma$. The value of $\left\langle\dot{a}^{(j)}\right\rangle_{t}$ for $\gamma=180^{\circ}$ has almost the same absolute value as that for $\gamma=0^{\circ}$ as seen from Table 1. The values of $\left\langle\dot{a}^{(j)}\right\rangle_{t}$ for $0^{\circ}<\gamma<90^{\circ}$ and $90^{\circ}<\gamma<180^{\circ}$ are given by a mixture of seasonal component and the diurnal component; it is difficult to define the error for mixture of two components because the sum of two 
components in some cases becomes very small due to the cancellation of positive and negative values (e.g. the case where $q_{\text {global }}=1 \times 10^{-2}, q_{\text {rev }}=0.2$, $q_{\text {rot }}=50$ and $\gamma=30^{\circ}$ in Table 1 ). The maximum error of the time rate of the change of the semimajor axis among our results is $24 \%$. Hence, the linear approximation (Vokrouhlický, 1999) is fairly good as far as the orbital evolution due to the Yarkovsky effect is concerned.

\section{Conclusions}

We have developed an iterative method for determining a time-dependent temperature distribution of a spherical body rotating itself and revolving around a star that irradiates the body. The nonlinear boundary condition with the radiative cooling that is the fourth power of the surface temperature is fully taken into account. We assumed that the body's surface is smooth and the thermal and the thermometric conductivities are constant in the body and remains constant for time. The main advantage of the present method compared to the previous works (Vokrouhlický, 1998, 1999) is that we can obtain a solution that satisfies the nonlinear boundary condition with an arbitrary accuracy by using large values of $n_{\max }$ and $\ell_{\max }$, using a large number of meshes for performing numerical integration of Eq. (28), and repeatedly using the above-mentioned recurrence formulas as many times as needed. The temperature distribution determined according to the iterative method is different from that according to the linear approximation; both the maximum and minimum temperatures for an iterative solution are lower than those for a linear solution. However, the rate of change in the semimajor axis due to the Yarkovsky effect determined by the linear solution (Vokrouh- 
lický, 1999) provides a fairly good approximation of that determined by the iterative method.

\section{Acknowledgments}

The authors are thankful to anonymous reviewers for their helpful comments. 


\section{Figure Captions}

Fig. 1. The coordinate system $(\tilde{x}, \tilde{y}, \tilde{z})$. The origin is at the center of the body. The axis of rotation is $\tilde{z}$, that has the obliquity $\gamma$ with respect to the orbital angular momentum of the body in the inertial system in which the star is at the origin. The direction of the star viewed from the body's center is in the $\tilde{x}-\tilde{z}$ plane and it is at an angle $\gamma$ from the $\tilde{x}$ axis at the summer solstice.

Fig. 2. The spherical coordinate system $(\tilde{r}, \theta, \phi)$. The origin is at the center of the body. The pole of this coordinate system is in the direction of the axis of rotation axis, $\tilde{z}$. The angle between the substellar point " $\star$ " at which the stellar direction is perpendicular to the body's surface and a point "P" with coordinates $(\tilde{R}, \theta, \phi)$ when viewed from the center of the body is $\Theta$.

Fig. 3. The coordinate system $\left(\tilde{x}^{\prime}, \tilde{y}, \tilde{z}^{\prime}\right)$. The origin and the $\tilde{y}$-axis are identical to those in the coordinate system $(\tilde{x}, \tilde{y}, \tilde{z})$ drawn in Figs. 1 and 2 . The $\tilde{z}^{\prime}$-axis is in the direction of the orbital angular momentum. The angle between the $\tilde{z}^{\prime}$-and $\tilde{z}$-axes is equal to the obliquity $\gamma$. The corresponding spherical coordinate system $\left(\tilde{r}, \theta^{\prime}, \phi^{\prime}\right)$, the pole of which is in the direction of $\tilde{z}^{\prime}$-axis, is shown. The angle between the substellar point " $\star$ " with coordinates $\left(\tilde{R}, \pi / 2, \omega_{\text {rev }} t\right)$ and a point "P" with coordinates $\left(\tilde{R}, \theta^{\prime}, \phi^{\prime}\right)$ when viewed from the center of the body is $\Theta$.

Fig. 4. The temperature distributions at the summer solstice in the case of $q_{\text {global }}=0.01, q_{\text {rot }}=50, q_{\text {rev }}=0.2$ and $\gamma=60^{\circ}$ determined by (a) the iterative method $\left(f_{\text {relax }}=0.5\right.$ and $\left.j=16\right)$ and (b) the linear approximation $\left(f_{\text {relax }}=1\right.$ and $\left.j=1\right)$. The north pole where $\theta=0^{\circ}$ is shown by "N", the south pole where $\theta=180^{\circ}$ by "S". The eastern end "E" and western 
end "W" on the equator of the Mollweide projection show an identical point with $(\theta, \phi)=\left(90^{\circ}, 180^{\circ}\right)$ and $\left(90^{\circ},-180^{\circ}\right)$, respectively. The substellar point marked by a star " $\star$ " is at $(\theta, \phi)=\left(30^{\circ}, 0^{\circ}\right)$.

Fig. 5. The temperature distributions at the autumn equinox for same values of parameters $q_{\text {global }}, q_{\text {rot }}, q_{\text {rev }}$ and $\gamma$ as Fig. 4. The symbols have same meanings as Fig. 4, but the coordinates of "E" , "W" and " $\star$ " at the autumn equinox are $(\theta, \phi)=\left(90^{\circ}, 270^{\circ}\right),\left(90^{\circ},-90^{\circ}\right)$ and $\left(90^{\circ}, 90^{\circ}\right)$. respectively.

Fig. 6. The temperature distributions at the summer solstice in the case of $q_{\text {global }}=1 \times 10^{-5}, q_{\text {rot }}=1.5, q_{\text {rev }}=6 \times 10^{-3}$ and $\gamma=60^{\circ}$ determined by (a) the iterative method $\left(f_{\text {relax }}=0.5\right.$ and $\left.j=41\right)$ and $(\mathrm{b})$ the linear approximation $\left(f_{\text {relax }}=1\right.$ and $\left.j=1\right)$. The symbols have same meanings as Fig. 4. The substellar point is at the same position as that in Fig. 4.

Fig. 7. The temperature distributions at the autumn equinox for same values of parameters $q_{\text {global }}, q_{\text {rot }}, q_{\text {rev }}$ and $\gamma$ as Fig. 6. The symbols have same meanings as Fig. 5. The substellar point is at the same position as that in Fig. 5. 


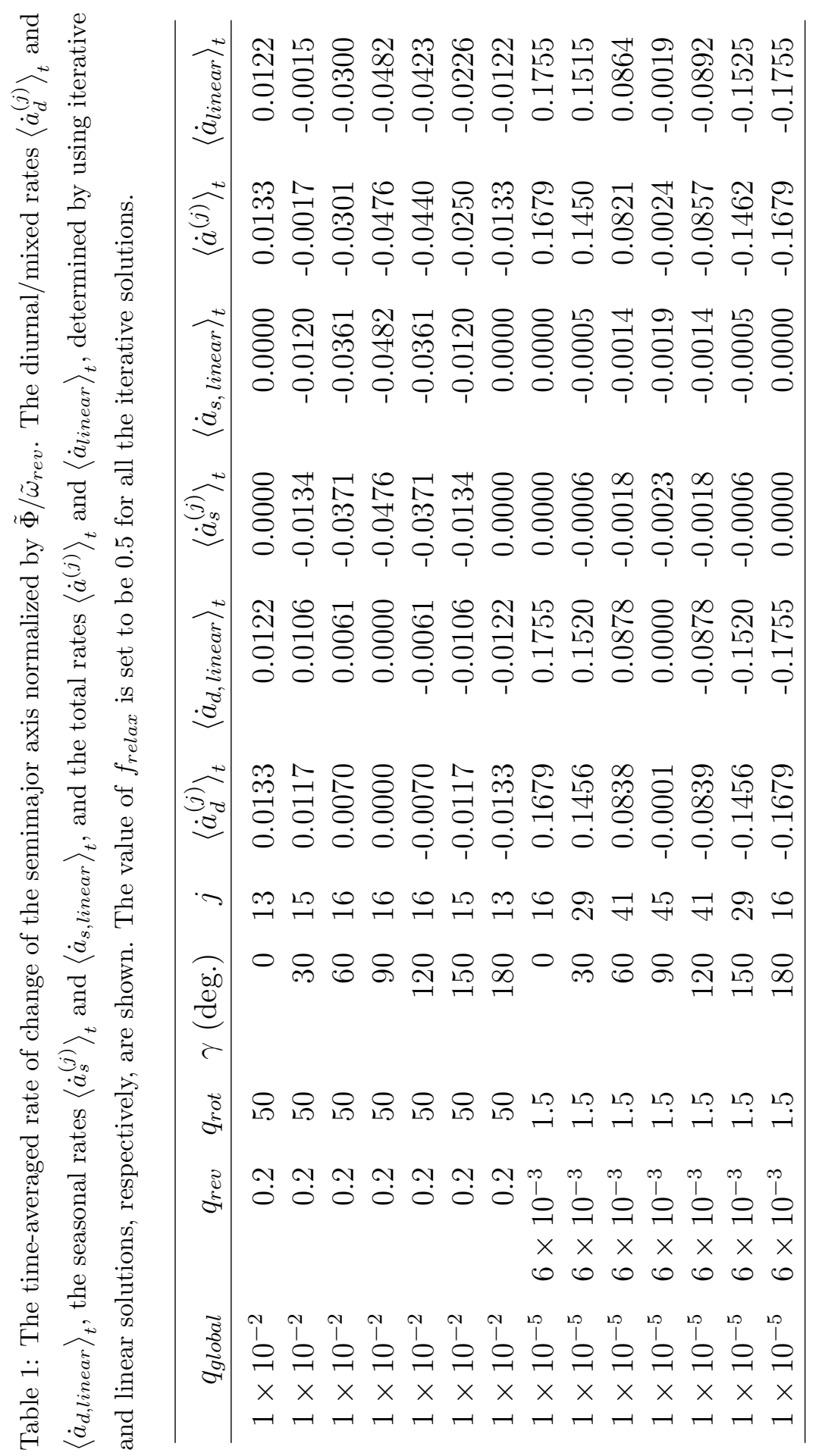




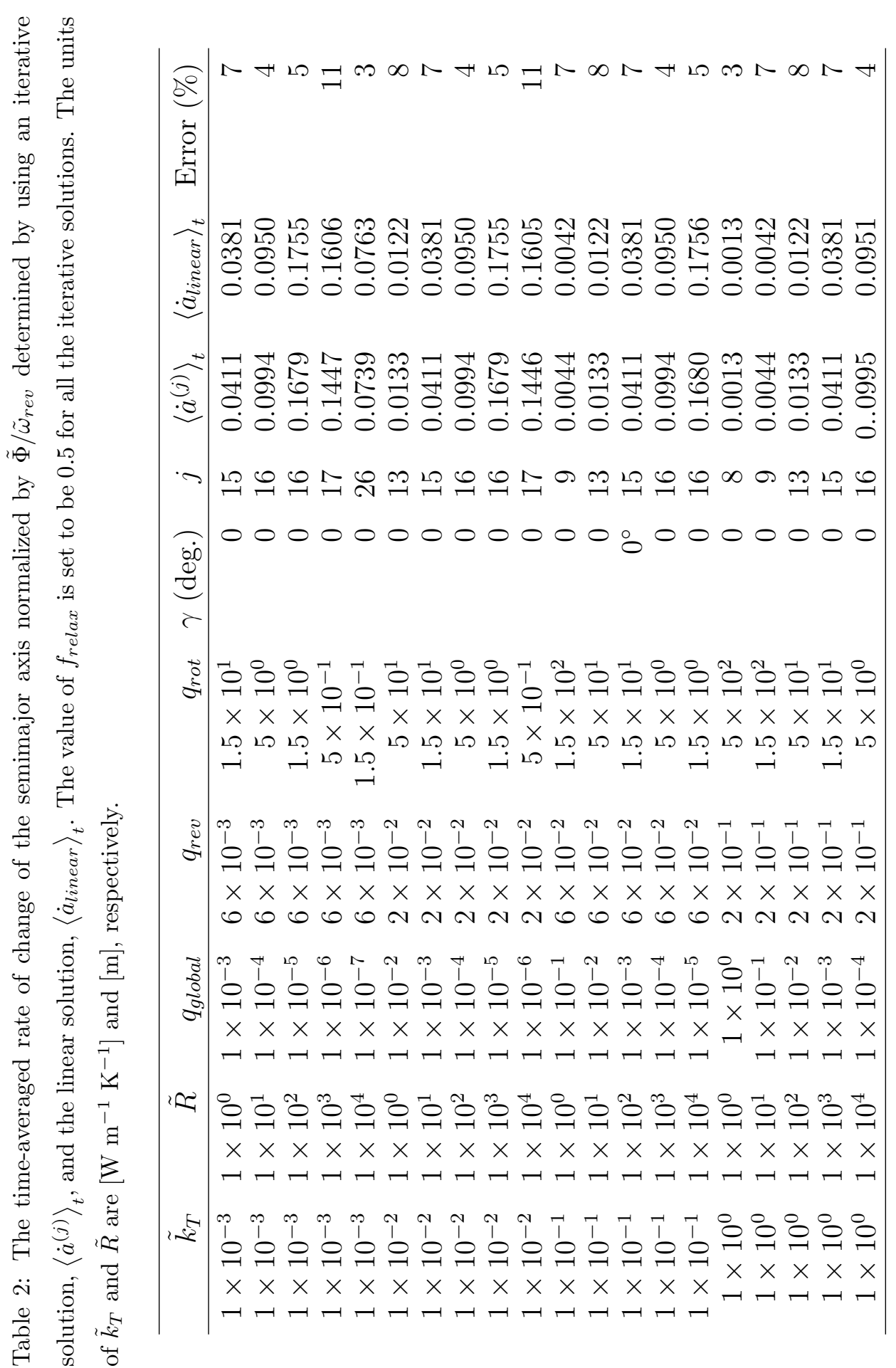




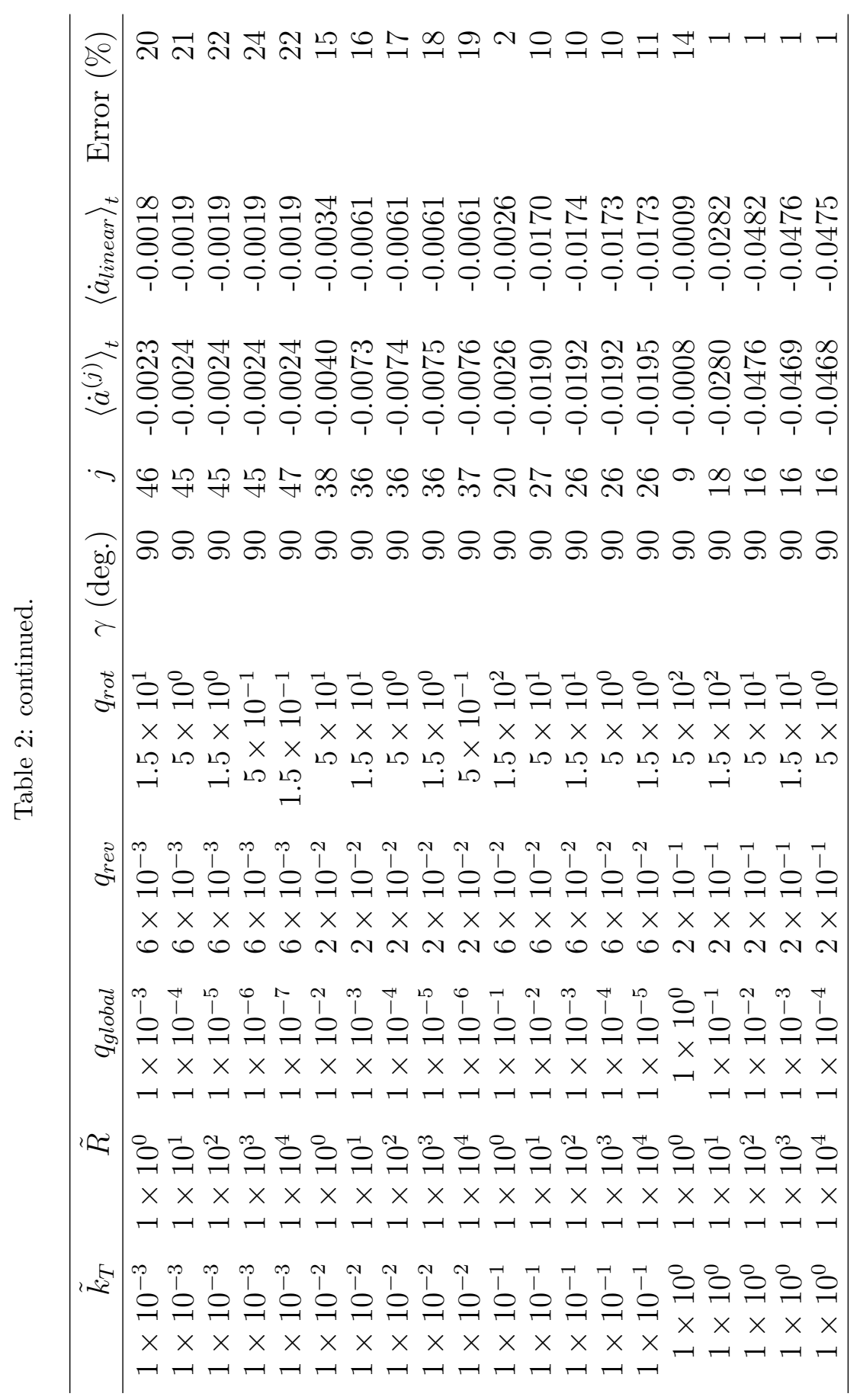




\section{References}

Arfken, G., 1970. Mathematical Methods for Physicists, second ed. Academic Press, New York.

Bischoff, A., Scott, E.R.D., Metzler, K., Goodrich, C.A., 2006. Nature and origins of meteoritic breccias. In: Lauretta, D.S., McSween Jr., H.Y. (Eds.) Meteorites and the Early Solar System II, University of Arizona Press, Tucson. pp. 679-712.

Bottke Jr., W.F., Vokrouhlický, D., Rubincam, D.P., Brož, M., 2002. The effect of Yarkovsky thermal forces on the dynamical evolution of asteroids and meteoroids. In: Bottke Jr. W.F., Cellino, A. Paolicchi, P., Binzel, R.P. (Eds.) Asteroids III, University of Arizona Press, Tucson. pp. 395-408.

Bottke Jr., W.F., Vokrouhlický, D., Rubincam, D.P., Nesvorný, D., 2006. The Yarkovsky and YORP effects: implications for asteroid dynamics. Annual Review of Earth and Planetary Sciences 34, 157-191.

Delbó, M., Harris, A.W., 2002. Physical properties of near-Earth asteroids from thermal infrared observations and thermal modeling. Meteoritics \& Planetary Science 37, 1929-1936.

Delbo, M., Michel, P., 2011. Temperature history and dynamical evolution of (101955) 1999 RQ 36: a potential target for sample return from a primitive asteroid. Astrophysical Journal Letters 728, L42.

Ghosh, A., McSween Jr., H.Y., 1998. A thermal model for the differentiation of asteroid 4 Vesta, based on radiogenic heating. Icarus 134, 187-206. 
Gladman, B., Michel, P., Froeschlé, C., 2000. The near-Earth object population. Icarus 146, 176-189.

Grimm, R.E., McSween Jr., H.Y., 1989. Water and the thermal evolution of carbonaceous chondrite parent bodies. Icarus 82, 244-280.

Kadono, T., Arakawa, M., Ito, T., Ohtsuki, K., 2009. Spin rates of fastrotating asteroids and fragments in impact disruption. Icarus 200, 694697.

La Spina, A., Paolicchi, P., Kryszczyńska, A., Pravec, P., 2004. Retrograde spins of near-Earth asteroids from the Yarkovsky effect. Nature 428, 400401.

Masiero, J.R., Mainzer, A.K., Grav, T., Bauer, J.M., Cutri, R.M., Dailey, J., Eisenhardt, P.R.M., McMillan, R.S., Spahr, T.B., Skrutskie, M.F., Tholen, D., Walker, R.G., Wright, E.L., DeBaun, E., Elsbury, D., Gautier IV, T., Gomillion, S. Wilkins, A., 2011. Main belt asteroids with WISE/NEOWISE. I. Preliminary albedos and diameters. Astrophysical Journal 741, 68 .

Michel, P., Delbo, M., 2011. Past thermal and orbital histories of 1999JU3 and 1999RQ36: two potential targets of sample return space missions to a primitive asteroid. Lunar and Planetary Science Conference XXXXII, Abst 1234 .

Miyamoto, M., Fujii, N., Takeda, H., 1981. Ordinary chondrite parent body: an internal heating model. In: Proceedings of Lunar and Planetary Sciences Conference, vol. 12B, 1145-1152. 
Morbidelli, A., Vokrouhlický, D., 2003. The Yarkovsky-driven origin of nearEarth asteroids. Icarus 163, 120-134.

Nakamura, T., Noguchi, T., Tanaka M., Zolensky, M.E., Kimura, M., Tsuchiyama, A., Nakato, A., Ogami, T., Ishida, H., Uesugi, M., Yada, T., Shirai, K., Fujiwara, A., Okazaki, R., Sandford, S.A., Ishibashi, Y., Abe, M., Okada, T., Ueno, M., Mukai, T., Yoshikawa, M., Kawaguchi, J., 2011. Itokawa dust particles: a direct link between S-type asteroids and ordinary chondrites. Science 333, 1113-1116.

Opeil, C.P., Consolmagno, G.J., Britt, D.T., 2010. The thermal conductivity of meteorites: new measurements and analysis. Icarus 208, 449-454.

Sekiguchi, T., Abe, M., Boehnhardt, H., Dermawan, B., Hainaut, O.R., Hasegawa, S., 2003. Thermal observations of MUSES-C mission target (25143) $1998 \mathrm{SF}_{36}$. Astronomy \& Astrophysics 397, 325-328.

Sekiya, M., Shimoda, A.A., Wakita, S., 2012. An iterative method for obtaining a nonlinear solution for the temperature distribution of a spinning spherical body irradiated by a central star. Planetary and Space Science $60,304-313$.

Vokrouhlický, D., 1998. Diurnal Yarkovsky effect as a source of mobility of meter-sized asteroidal fragments I. Linear theory. Astronomy \& Astrophysics 335, 1093-1100.

Vokrouhlický, D., 1999. A complete linear model for the Yarkovsky thermal force on spherical asteroid fragments. Astronomy \& Astrophysics 344, 362366. 
Vokrouhlický, D., Farinella, P., 1999. The Yarkovsky seasonal effect on asteroidal fragments: a nonlinearized theory for spherical bodies. Astronomical Journal 118, 3049-3060.

Vokrouhlický, D., Brož, M., 1999. An improved model of the seasonal Yarkovsky force for regolith-covered asteroid fragments. Astronomy \& Astrophysics 350, 1079-1084.

Vokrouhlický, D., Brož, M., Morbidelli, A., Bottke, W.F., Nesvorný, D., Lazzaro, D., Rivkin, A.S., 2006a. Yarkovsky footprint in the Eos family. Icarus 182, 92-117.

Vokrouhlický, D., Brož, M., Bottke, W.F., Nesvorný, D., Morbidelli, A., 2006b. Yarkovsky/YORP chronology of asteroid families. Icarus 182, 118142.

Wakita, S., Sekiya, M., 2011. Thermal evolution of icy planetesimals in the solar nebula. Earth Planets Space 63, 1193-1206.

Wigner, E.P., 1959. Group Theory and Its Application to the Quantum Mechanics of Atomic Spectra. Academic Press, New York (trans. Griffin, J.J.).

Young, E.D., Zhang, K.K., Schubert, G., 2003. Conditions for pore water convection within carbonaceous chondrite parent bodies - implications for planetesimal size and heat production. Earth and Planetary Science Letters 213, 249-259.

Yomogida, K., Matsui, T., 1983. Physical properties of ordinary chondrites. Journal of Geophysical Research 88, 9513-9533. 


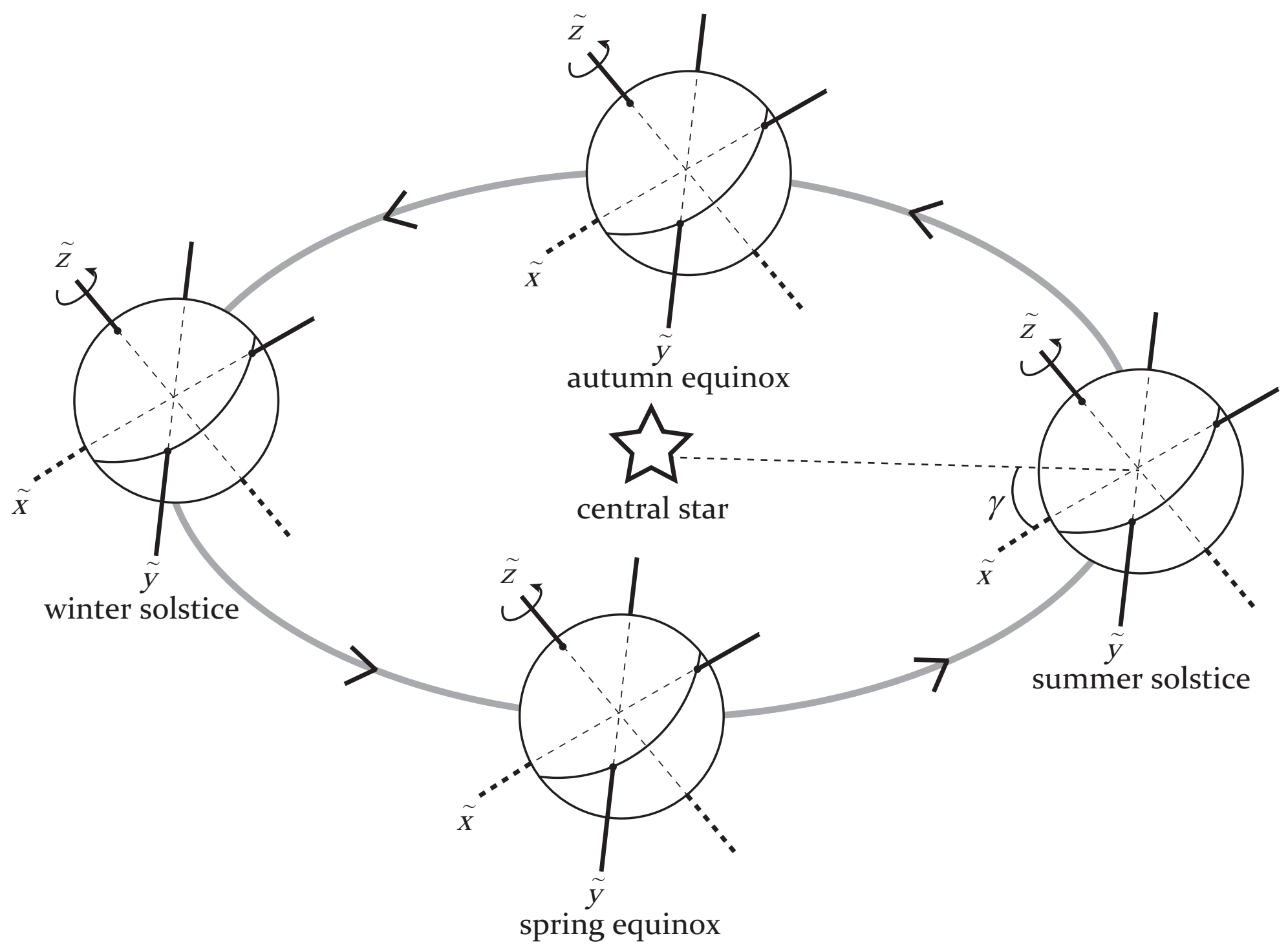

Fig. 1 


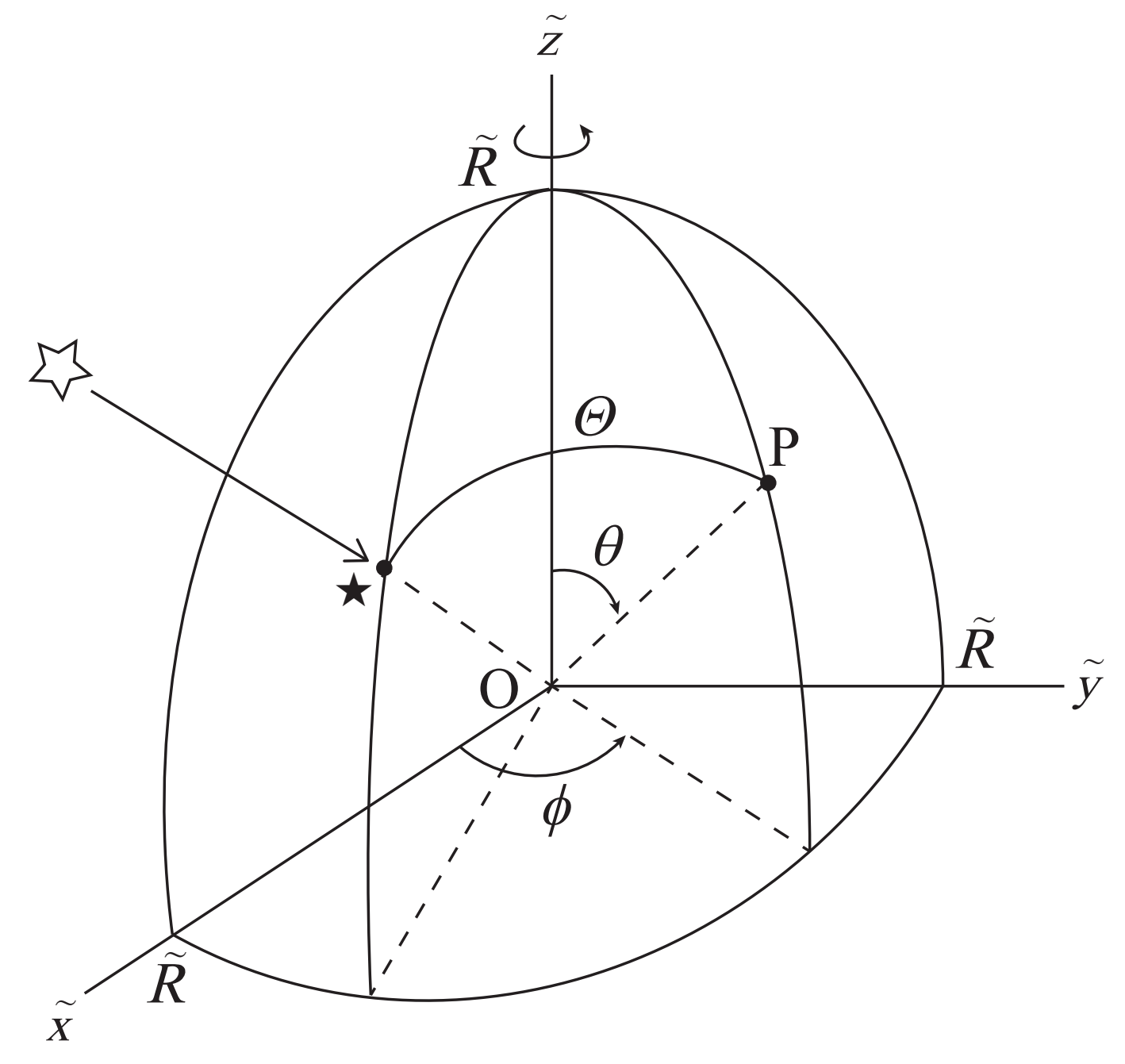

Fig. 2 


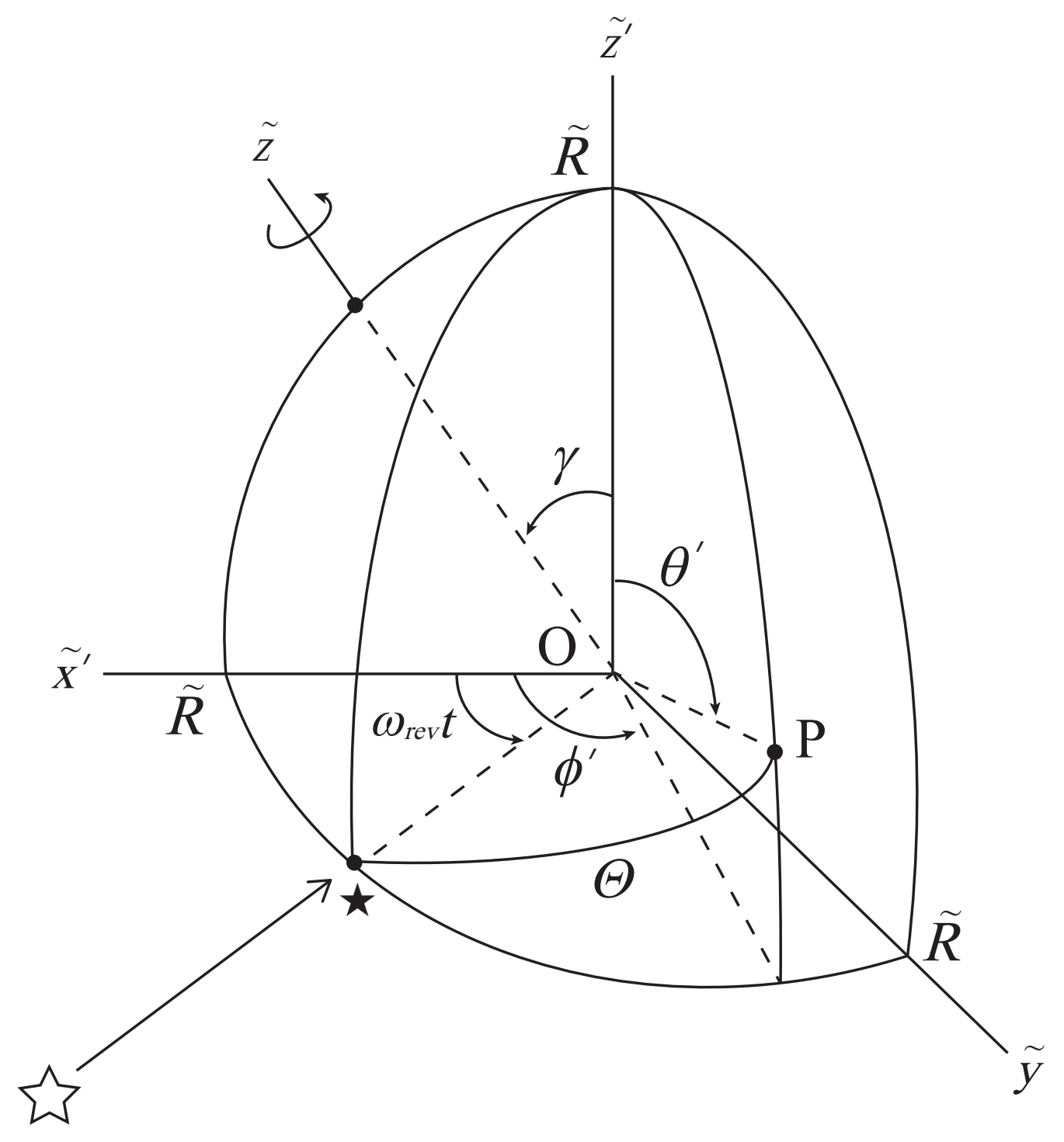

Fig. 3 
(a)

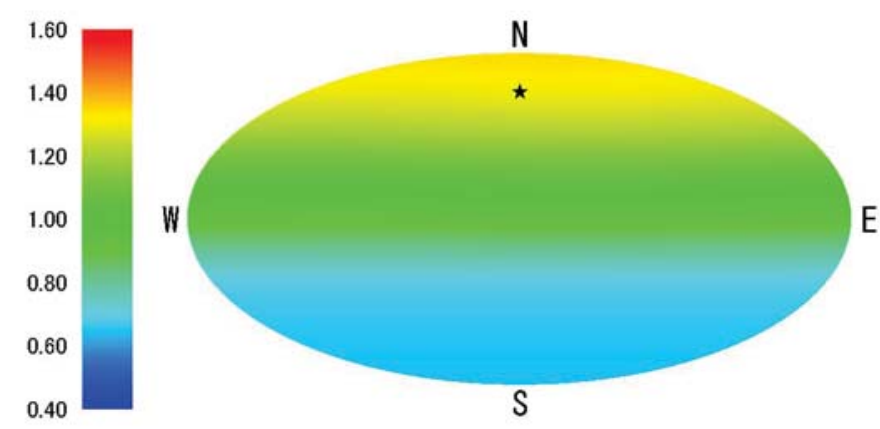

(b)

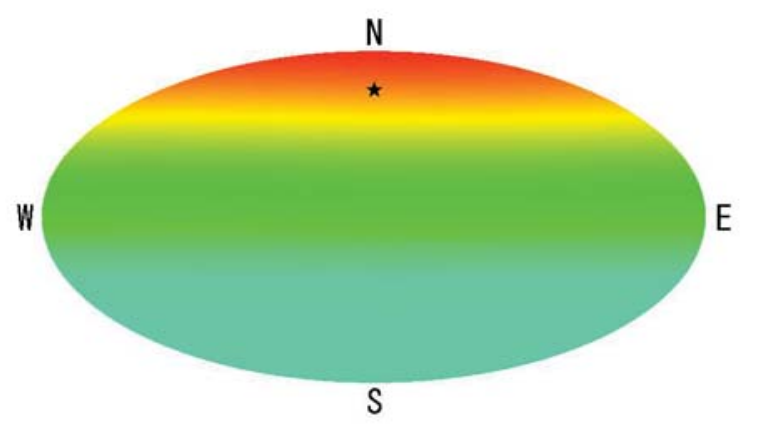

Fig. 4 

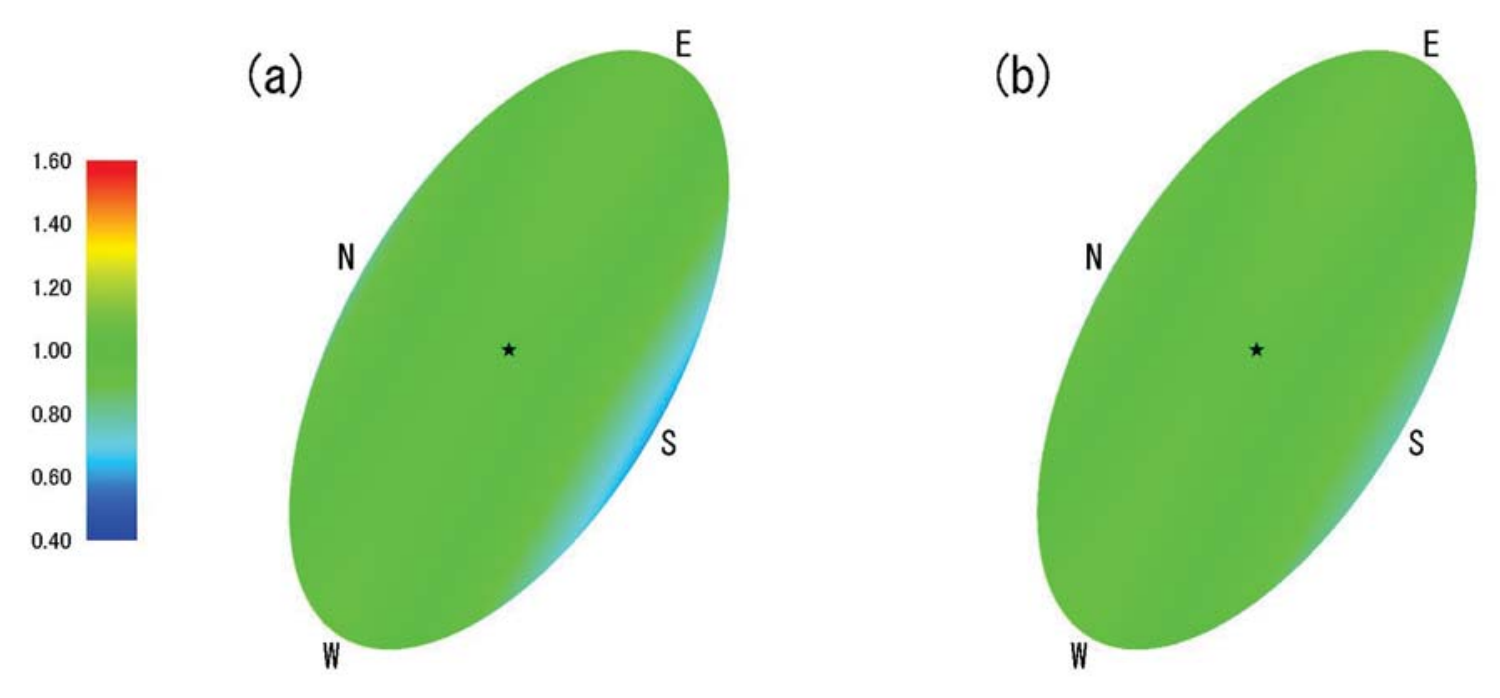

Fig. 5 
(a)

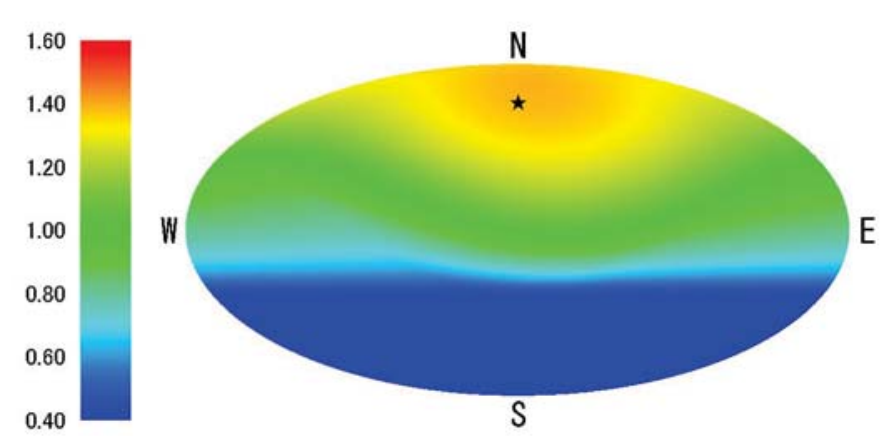

(b)

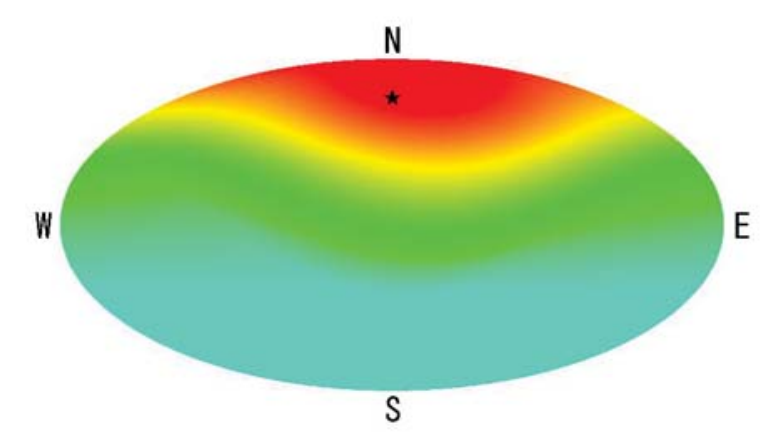

Fig. 6 

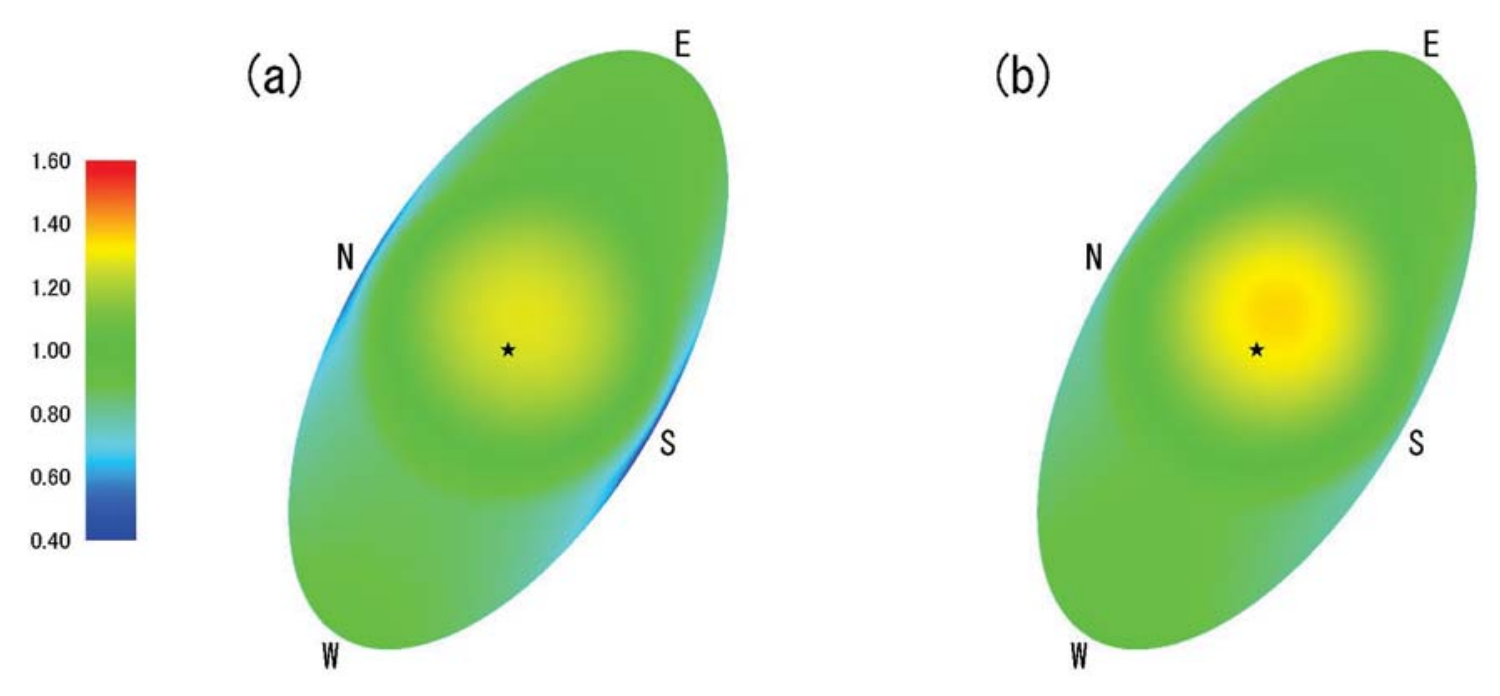

Fig. 7 